\title{
NEWTON POLYHEDRA, A NEW FORMULA FOR MIXED VOLUME, PRODUCT OF ROOTS OF A SYSTEM OF EQUATIONS
}

\section{A. Khovanskii}

\begin{abstract}
We generalize Vieta formula for the product of roots of a polynomial to the multidimensional case. We compute in the group $\left(\mathbb{C}^{*}\right)^{n}$ the product of all roots of a system of $n$ polynomial equations with sufficiently general Newton polyhedra. We present two different formulas for this product. In the first formula we use the so called Parshin symbols, in the second formula we use derivatives of the mixed volume with respect to vertices of all polyhedra. Both formulas employ certain combinatorial coefficients which characterize the relative location of Newton polyhedra at each vertex of their Minkowski sum. The technique of these coefficients is essential for our work. Using this technique we also prove a new formula for mixed volumes.
\end{abstract}

To Vladimir Igorevich Arnold

\section{Introduction}

1. The idea of this work came to me in the summer of 1995 in Paris, when I was staying with V. I. Arnold. I am grateful to Vladimir Igorevich for his hospitality and for stupendous walks around Paris and its vicinities.

2. According to the classical Vieta formula, the product of the nonzero roots of an equation $a_{n} x^{n}+\cdots+a_{k} x^{k}=0$ with $a_{n} \neq 0, a_{k} \neq 0$ is equal to the number $(-1)^{n-k} a_{k} a_{n}^{-1}$. In this article we generalize the Vieta formula to the multidimensional case. More precisely, we compute in the group $\left(\mathbb{C}^{*}\right)^{n}$ the product of all the roots of the system of equations

$$
P_{1}(x)=\cdots=P_{n}(x)=0, \quad x \in\left(\mathbb{C}^{*}\right)^{n}
$$

whose Newton polyhedra $\Delta_{1}, \ldots, \Delta_{n}$ are developed (see $\S 1$ ), which means that they are located sufficiently generally with respect to each other.

The geometrical meaning of being developed is especially clear in the twodimensional case: two polygons on a plane are developed if and only if they do not have parallel sides with identically directed outer normals.

For the generalization of the Vieta formula the condition of developed polyhedra is essential. If it is not satisfied, then the problem of finding the product of all the

This work was completed with the partial support of Canadian Grant OGP0156833 and grant N 95-011-8701 of Russian Fond of Fundamental Research. 
roots of a system of equations turns out to be just as difficult as the problem of finding the multidimensional resultants (see theorem $1, \S 6){ }^{1}$

The Newton polygon of the polynomial $P(x)=a_{n} x^{n}+\cdots+a_{k} x^{k}$ is a segment $I(n, k)$ on the real line with vertices $n$ and $k$, where $n>k \geq 0$. The product of the nonzero roots of the polynomial $P$ is equal, up to a sign, to the monomial $a_{k} a_{n}^{-1}$ in the coefficients $a_{n}, a_{k}$ at the vertices $n$ and $k$ of the Newton polyhedron $I(n, k)$ of the polynomial $P$. The coefficient $a_{n}$ enters in this monomial to the power given by the value, taken with opposite sign, of the derivative of the length of the segment $I(n, k)$ by the vertex $n$, i.e. to the power minus one. The coefficient $a_{k}$ enters in this monomial to the power also given by the value, taken with opposite sign, of the derivative of the length of the segment $I(n, k)$ by the vertex $k$, i.e. to the power one.

It turns out that this is the case in many dimensions as well. Up to a sign every component of the product of the roots of a system of equations with developed Newton polyhedra is equal to a certain monomial in the coefficients of equations corresponding to the vertices of the Newton polyhedra. Further, the coefficient at each vertex enters in these monomials to vector power equal to the value, taken with coefficient $(-n !)$, of the derivative of the mixed volume of the Newton polyhedra by this vertex (see theorem 2 from $\S 11$ ).

3. The Vieta formula also has a completely different interpretation connected with Weil's theorem. First let us recall Weil's theorem (see, for example, [8]). Let $X$ be a complex algebraic curve, and $f$ and $g$ be two nonzero holomorphic functions on $X$. With each point $a \in X$ is associated the so-called the Weil symbol $[f, g]_{a}$. Here is its definition. Let $u$ be a local parameter about the point $a, u(a)=0$, and let $f=c_{1} u^{k_{1}}+\ldots$ and $g=c_{2} u^{k_{2}}+\ldots$ be the leading terms of the expansion of the functions $f$ and $g$ at the point $a$. The Weil symbol $[f, g]_{a}$ is the number $(-1)^{k_{1} k_{2}} c_{1}^{k_{2}} c_{2}^{-k_{1}}$ (let us point out that sometimes the sign in the Weil symbol is chosen differently, instead of $(-1)^{k_{1} k_{2}}$ it is sometimes set equal to $\left.(-1)^{k_{1}+k_{2}+k_{1} k_{2}}\right)$. For all the points on the curve $X$, except for a finite number of them, the Weil symbol is equal to one. The following holds:

Weil's theorem.

$$
\prod_{a \in X}[f, g]_{a}=1
$$

Let us apply Weil's theorem in the case that the curve $X$ coincides with the Riemann sphere, the function $f$ is equal to the coordinate function $x$, and the function $g$ is equal to a polynomial $P$. We will get

$$
\prod x(a)=[x, P]_{0}^{-1}[x, P]_{\infty}^{-1},
$$

\footnotetext{
${ }^{1}$ During my visit to the City University of Hong Kong in 1999 I learned that a formula for the product of roots that uses multidimensional resultants was found independently in the following papers:

1. E. Cattani, A. Dickenstein and B. Sturmfels, Residues and Resultants, J. Math. Sci. Univ. Tokyo 5 (1998), no. 1, 119-148.

2. J.M. Rojas, Toric Laminations, Sparse Generalized Characteristic Polynomials, and a Refinement of Hilbert's Tenth Problem, Foundations of Computational Mathematics, selected papers of a conference, IMPA, Rio de Janeiro, 1997, Springer-Verlag, Berlin-New York, 1999, pp. $369-381$.
} 
where the product is taken over all nonzero roots $a$ of the polynomial $P$. This formula coincides with the Vieta formula.

Parshin-Kato theory gives a far-reaching generalization of Weil's theorem. In this theory to $(n+1)$ meromorphic functions on an $n$-dimensional algebraic manifold $X$ and a flag of submanifolds in the manifold $X$ is associated the so-called Parshin symbol which generalizes the Weil symbol. According to Parshin-Kato theory, the product of Parshin symbols over certain flags of submanifolds also turns out to by equal to one (see [9], [10], [12]).

Let us turn to a different interpretation of the multidimensional generalization of the Vieta formula. Let $M\left(P_{1}, \ldots, P_{n}\right)$ be the product in the group $\left(\mathbb{C}^{*}\right)^{n}$ of the roots of the system of equations (1). The computation of the point $M\left(P_{1}, \ldots, P_{n}\right)$ in the group $\left(\mathbb{C}^{*}\right)^{n}$ is equivalent to the computation of the value $\chi\left(M\left(P_{1}, \ldots, P_{n}\right)\right)$ of each character $\chi:\left(\mathbb{C}^{*}\right)^{n} \rightarrow \mathbb{C}^{*}$ at this point.

Let $\Delta$ be the Minkowski sum of the polyhedra $\Delta_{i}, \Delta=\Delta_{1}+\cdots+\Delta_{n}$. With each vertex $A$ of the polyhedron $\Delta$ is associated an integer - the combinatorial coefficient $C_{A}$ of the vertex $A$ - characterizing the mutual position of the polyhedra $\Delta_{1}, \ldots, \Delta_{n}$ in the space. In this paper the combinatorial coefficients (see $\S \S 1-3$ ) play an important role.

With each vertex $A$ of the polyhedron $\Delta$ it is possible to associate a number $\left[P_{1}, \ldots, P_{n}, \chi\right]$, which we will call the Parshin symbol of the functions $P_{1}, \ldots, P_{n}, \chi$ at the vertex $A$ of the polyhedron $\Delta$ (see $\S 11$ ).

Let us cite another expression for the product of the roots of a system of equations. Namely the following equality holds (see theorem $1 \S 11$ ):

$$
\chi\left(M\left(P_{1}, \ldots, P_{n}\right)\right)=\prod_{A \in \Delta}\left[P_{1}, \ldots, P_{n}, \chi\right]_{A}^{(-1)^{n} C_{A}} .
$$

Here the product is taken over all the vertices $A$ of the polyhedron $\Delta$. The equality (3) is analogous to the interpretation of Vieta's theorem (2) with the help of Weil's theorem. It should be explainable in the framework of Parshin-Kato theory. Our proof of the equality (3), however, is elementary and does not require this theory.

4. The results of this paper are connected to the results of the work [4], although they are independent of each other. Several words about the work [4]. In [4] there is an explicitly calculated formula for the Grothendieck residues of the form

$$
\omega=\frac{Q}{P_{1} \ldots P_{n}}\left(\frac{d x_{1}}{x_{1}} \wedge \cdots \wedge \frac{d x_{n}}{x_{n}}\right)
$$

over all the roots of the system of equations (1), where $Q$ is an arbitrary Laurent polynomial. If we let $Q=x_{1} \ldots x_{n} T \operatorname{det}\left(\frac{\partial P}{\partial x}\right)$, where $T$ is an arbitrary Laurent polynomial, then the sum of the Grothendieck residues of the form $\omega$ will be equal to the sum of the values of the Laurent polynomial $T$ over the roots of the system of equations (1).

The formula for the sum of the Grothendieck residues from [4] is of the form

$$
\sum\left[\frac{Q}{P_{1}, \ldots P_{n}}\right]_{a}=\sum_{A \in \Delta}(-1)^{n} C_{A} \operatorname{res} \omega_{A}
$$


where the summation on the left hand side of the equality is taken over all the roots of the system of equations (1), and the summation on the right hand side of the equality is taken over all the vertices $A$ of the polyhedron $\Delta ; \operatorname{res} \omega_{a}$ is the residue of the form $\omega$ at the vertex $A$ (see [4]), and $C_{A}$ is the combinatorial coefficient at the vertex $A$.

The formula (3) is similar to the formula (4). I hope to return to this range of questions and explain the reason behind the similarity of these formulas.

5. One of the consequences of the work [4] is a new formula for the mixed volume of a developed collection of integer-valued polyhedra. It is obtained by summing over the roots of the system a polynomial identically equal to one. This sum than equals the number of roots of the system, which by Bernshtein's theorem [1] equals $n$ ! multiplied by the mixed volume of the Newton polyhedra: $n ! \operatorname{Vol}\left(\Delta_{1}, \ldots, \Delta_{n}\right)$. Using the formula (4) we obtain

$$
n ! \operatorname{Vol}\left(\Delta_{1}, \ldots \Delta_{n}\right)=\sum_{A \in \Delta}(-1)^{n} C_{A} \operatorname{det}\left(A_{1}, \ldots, A_{n}\right) .
$$

Here the summation is taken over all the vertices $A$ of the polyhedron $\Delta$, and $A_{1}, \ldots, A_{n}$ are vertices of the polyhedra $\Delta_{1}, \ldots, \Delta_{n}$ that $A_{1}+\cdots+A_{n}=A$. In this way, the new formula for mixed volumes was proven in [4] by methods of algebraic geometry but only for integer-valued polyhedra. In spite of some progress (see [5]), efforts to generalize the formula (5) to the case of non-integral polyhedra have not previously been successful. Here we fill in this gap (see $\S 4$ ).

6. A couple of words about the arrangement of material in this paper.

Paragraphs 1-4 are devoted to polyhedral geometry. In $\S 1$ we introduce the combinatorial coefficients of a developed collection of polyhedra. In $\S 2$ we discuss subdivisions of several polyhedra and compatibility of these subdivisions. In $\S 3$ we generalize the definition of the combinatorial coefficients to the case of compatible subdivisions of polyhedra. In $\S 4$ we prove a new formula for the mixed volume of a collection of polyhedra and compute the derivatives of the mixed volume by the vertices of the polyhedra. After this we turn to algebraic geometry.

Our method to study the product of the roots of a system of equations consists of the following. In the system we introduce an extra parameter $t$ so that when $t \rightarrow \infty$ the roots of the system split into groups of roots having different asymptotics. Furthermore the dependence on the parameter $t$ is chosen so that each group of roots with the same asymptotics would be the simplest possible (see theorem 2 from $\S 7$ ), and so that the dependence of the product on the parameter $t$ would be possible to control (see theorem 3 from $\S 5$ ).

A similar idea was used in the work of D. Bernshtein [1], in which he computes the number of roots of a system of equations; in the work of O.Ya. Viro [11], in which he constructs nontrivial examples of real algebraic manifolds; and in the work of I.M. Gelfand, A.M. Zelvinski and M. Kapranov [3], in which they study multidimensional discriminants.

The simplest systems, which cannot be further simplified, are systems of binomial equations. These systems can be solved explicitly. The product of the roots of such systems is calculated in $\S 8$. The determination of signs in this computation oriiginally gave me the most difficulty, but turns out to be very simple: in $\S 9$ we investigate in detail linear algebra over the field $\mathbb{Z} / 2 \mathbb{Z}$ which is connected to the 
computation of these signs. $\S 10$ is also devoted to systems of binomial equations: the product of roots of such systems can be written in an especially convenient way. In $\S 11$ we combine our calculations with the technique of combinatorial coefficients from $\S \S 1-4$ and conclude the proof of the multidimensional generalization of the Vieta formula.

7. I am grateful to my wife T.V. Belokrinitskaya for her help with the work on this paper, and to A. Ivrii for his help in preparing this paper for publication. A number of results of this work were obtained during my visit to the Ecole Normale Supérieure (France) in 1995 and in 1996. I could finish this paper thanks to reduction of professorial load, which was offered to me for scientific research in 1997-1998 by the University of Toronto.

\section{$\S 1$. Characteristic maps, critical vertices, and developed sets of polyhedra}

In this paragraph we discuss the combinatorial coefficients - integral invariants characterizing the mutual placement of $n$ polyhedra in a $n$-dimensional space. The technique of combinatorial coefficients plays a central role in this paper.

Let $\Delta_{1}, \ldots, \Delta_{n}$ be convex polyhedra, lying in some real linear space, and let $\Delta$ be their Minkowski sum. Each face $\Gamma$ of the polyhedron $\Delta$ can be uniquely represented as a sum

$$
\Gamma=\Gamma_{1}+\cdots+\Gamma_{n}
$$

of faces $\Gamma_{i}$ of the polyhedra $\Delta_{i}$. The representation (1) will be called the decomposition of the face $\Gamma$ and the face $\Gamma_{i} \subset \Delta_{i}$ will be called the $i$-th component of the decomposition of the face $\Gamma$. The face $\Gamma$ will be called locked if among the components of its decomposition there is at least one vertex.

Let $K$ be the union of all the locked faces of the polyhedron $\Delta$. A map

$$
f: K \rightarrow \partial \mathbb{R}_{+}^{n}
$$

of the set $K$ into the boundary of the positive octant will be called characteristic if the component $f_{i}$ of the map $f=\left(f_{1}, \ldots, f_{n}\right)$ vanishes on and only on the faces $\Gamma$ from the set $K$ for which the $i$-th component of the decomposition is a point (a vertex of the polyhedron $\Delta_{i}$ )

Lemma 1. 1. Characteristic maps exist.

2. For a characteristic map the preimage of the center of coordinates is precisely the set of all the vertices of the polyhedron $\Delta$.

3. The set of all characteristic maps is convex.

Proof. 1 . The set $M_{i}$ of the faces of the polyhedron $\Delta$, for which the $i$-th component of decomposition is a vertex, with each face $\Gamma$ also contains all the faces belonging to its boundary $\partial \Gamma$. Therefore there exists a nonnegative function $f_{i}$ on the set $K$ which vanishes precisely on the faces in the set $M_{i}$. The map $f$ with components $f_{1}, \ldots, f_{n}$ maps the set $K$ into the boundary of the positive octant because by definition all the faces of the polyhedron $\Delta$ in the set $K$ are locked.

2. Only for the vertices of the polyhedron $\Delta$ all the components of the decomposition are vertices of the polyhedra $\Delta_{i}$. 
3. If $f$ and $h$ are characteristic maps, then for every $t, 0 \leq t \leq 1$, the map $f_{t}=t f+(1-t) h$ is also characteristic. In particular $f_{t}$ is a map into the boundary of the positive octant.

Remark. The characteristic map can be chosen piecewise linear. Moreover, using barycentric subdivision of the polyhedron $\Delta$, it is possible to construct a characteristic map explicitly.

We will call a map $h: K \rightarrow \partial \mathbb{R}_{+}^{n}$ almost characteristic if

1) the component $h_{i}$ of the map $h=\left(h_{1}, \ldots, h_{n}\right)$ vanishes on the faces $\Gamma$ from the set $K$ for which the $i$-th component of the decomposition is a point;

2) The map $h$ maps into the center of coordinates only the vertices of the polyhedron $\Delta$.

The components of almost characteristic maps can vanish on bigger sets than the corresponding components of the characteristic map. Almost characteristic maps belong to the closure of the convex set of characteristic maps. It is easy to check the following

Lemma 2. Let $f$ be a characteristic map and let $h$ be an almost characteristic map. Then the map $h_{t}=t f+(1-t) h$ with $0<t \leq 1$ is characteristic.

A vertex of the polyhedron $\Delta$ will be called critical if all the proper faces of the polyhedron $\Delta$ adjacent to this vertex are locked. In other words, a vertex will be called critical if it belongs to the interior of the set $K \cap \partial \Delta$ in the topology of the boundary of the polyhedron $\Delta$.

Assume further that the polyhedron $\Delta$ lies in a linear space $L$ of dimension $n$; let us fix the orientations of the spaces $L$ and $\mathbb{R}^{n}$.

Definition. The combinatorial coefficient $C_{A}$ of a critical vertex $A$ of the polyhedron $\Delta$ is the local degree of the germ of the map

$$
f:(\partial \Delta, A) \rightarrow\left(\partial \mathbb{R}_{+}^{n}, 0\right)
$$

of the restriction of a characteristic map on the boundary of the polyhedron $\Delta$.

Lemma 3. 1. The combinatorial coefficient is well-defined, i.e. it does not depend on the choice of the characteristic map.

2. The local degree of the germ of an almost characteristic map at a critical vertex of the polyhedron is equal to the combinatorial coefficient of this vertex.

Proof. The first part follows by lemma 1, and the second part follows by lemma 2 .

We will say that a collection of $n$ polyhedra $\Delta_{1}, \ldots, \Delta_{n}$ in a linear space $L$ is developed if all the faces of the polyhedron $\Delta$ are locked. In other words, the collection of polyhedra is developed if the set $K$ contains the boundary of the polyhedron $\Delta$. Thus the combinatorial coefficient is defined at every vertex of the sum polyhedron.

Let us consider one simple but important example. Let $I_{1}, \ldots, I_{n}$ be $n$ transversal segments in $\mathbb{R}^{n}$, and let $\Delta=I_{1}+\cdots+I_{n}$ be their Minkowski sum. Each vertex $A$ of the parallelepiped $\Delta$ is the sum of certain vertices $A_{i}$ of segments $I_{i}$ : $A=$ $A_{1}+\cdots+A_{n}$. For each segment $I_{i}$ fix one of its vertices $l_{i}$ which we will call left; the second vertex will be called right and it has the form $h_{i}=l_{i}+a_{i}$, where $a_{i}$ is a vector from the first vertex to the second. 
Theorem 1. The combinatorial coefficient at a vertex $A=A_{1}+\cdots+A_{n}$ of the parallelepiped $\Delta=I_{1}+\cdots+I_{n}$ is equal to $(-1)^{H(A)} \operatorname{sign} \operatorname{det}\left(a_{1}, \ldots, a_{n}\right)$, where $H(A)$ is the number of points $A_{i}$ being the right vertices of segments $I_{i}$, and $a_{i}$ is the vector from the left vertex of $I_{i}$ to the right vertex.

Proof. First consider $n$ usually situated segments $I_{1}, \ldots I_{n}$ in the space $\mathbb{R}^{n}$ equiped with standard orientation. Namely, define the segment $I_{i}$ by the following relations: the point $x_{1}, \ldots, x_{n}$ belongs to $I_{i}$ if and only if $x_{j}=0$ for $i \neq j$ and $-1 \leq x_{i} \leq 1$. The Minkowski sum of these segments is a cube $\Delta$ defined by the relations $\left(x_{1}, \ldots, x_{n}\right) \in \Delta \Leftrightarrow\left|x_{i}\right| \leq 1$. Consider the map $f$ of $\Delta$ in $\mathbb{R}_{+}^{n}$ defined by $f\left(x_{1}, \ldots, x_{n}\right)=1-\left|x_{1}\right|, \ldots, 1-\left|x_{n}\right|$. With the help of this map we can compute the combinatorial coefficients. It is easy to see that the local degree of the restriction of the map $f$ on the boundary $\partial \Delta$ of the cube into the boundary of the positive octant $\partial \mathbb{R}_{+}^{n}$ is computed as follows. The local degree of the map at a vertex $A$ is equal to $(-1)^{H(A)}$, where $H(A)$ is the number of coordinates of the vertex $A$ equal to +1 . (Every coordinate of any vertex $A$ of the cube $\Delta$ is equal either to +1 or to -1.) This proves the theorem in the considered case.

Now let $I_{1}, \ldots, I_{n}$ be transversal segments the midpoints of which are situated in the center of coordinates. Consider a linear transformation of the space $\mathbb{R}^{n}$ which sends the $i$-th vector of the standard basis of the space $\mathbb{R}^{n}$, to the vector $a_{i}$. This transformation preserves orientation if $\operatorname{det}\left(a_{1}, \ldots, a_{n}\right)$ is positive, and reverses the orientation in the opposite case. This transformation reduces the case to the one previously considered.

Finally, a parallel translation of segments $I_{i}$ results in simply translating the Minkowski sum of these segments. So theorem 1 is proven in the general case.

For $i=1, \ldots,(n-1)$ let us fix certain vertices $A_{i}$ of the polyhedra $\Delta_{i}$. Consider the set $V\left(A_{1}, \ldots, A_{n-1}\right)$ of the vertices of the polyhedron $\Delta$ for which the vertices $A_{1}, \ldots, A_{n-1}$ are the components of the decomposition. In other words, a vertex $A$ of the polyhedron $\Delta$ belongs to the set $V\left(A_{1}, \ldots, A_{n-1}\right)$ if there exists a vertex $A_{n}$ of the polyhedron $\Delta_{n}$ such that $A=A_{1}+\cdots+A_{n-1}+A_{n}$.

Theorem 2. For every developed collection of polyhedra $\Delta_{1}, \ldots, \Delta_{n}$ and for every choice of vertices $A_{1}, \ldots, A_{n-1}$ the following statement holds. The sum of the combinatorial coefficients of the vertices $A$ of the polyhedron $\Delta$ belonging to the set $V\left(A_{1}, \ldots, A_{n-1}\right)$ is equal to 0 , i.e.

$$
\sum_{A \in V\left(A_{1}, \ldots, A_{n-1}\right)} C_{A}=0
$$

Proof. Let us fix a characteristic map and denote by $f: \partial \Delta \rightarrow \partial \mathbb{R}_{+}^{n}$ its restriction on the boundary of the polyhedron $\Delta$. Consider the vertical ray $x_{1}=\cdots=x_{n-1}=0$, $x_{n} \geq 0$, lying in the boundary of the positive octant $\mathbb{R}_{+}^{n}$. Its preimage under the map $f$ consists of the union $M$ of all the faces $\Gamma$ of the polyhedron $\Delta$ such that their $i$-th components of the decomposition for $i=1, \ldots,(n-1)$ are vertices. A subset $M_{0}$ of the set $M$ consisting of the faces for which these components are the fixed vertices $A_{1}, \ldots, A_{n-1}$ is isolated. In other words, it is possible to choose an open neighbourhood $U$ of the set $M_{0}$ such that its closure does not contain any other points from the set $M$. On the boundary of this neighbourhood the function $f_{1}+\cdots+f_{n-1}$ reaches a minimum value $C$, moreover this minimum value is positive. 
Denote by $W_{C}$ an unbounded region in the boundary of the positive octant which contain the vertical ray and whose points satisfy the inequality $x_{1}+\cdots+x_{n-1}<C$. Denote by $U_{C}$ the set $U \cap f^{-1}\left(W_{C}\right)$.

The map $f: U_{C} \rightarrow W_{C}$ is a proper map, so the notion of degree of $f$ makes sense. On one hand, this degree equals 0 since the component $f_{n}$ of the function $f$ is bounded on the polyhedron $\Delta$ so the points on the vertical ray sufficiently far from the origin do not have a preimage at all. On the other hand, this degree equals to the number of preimages of the center of coordinates, calculated counting the multiplicities, i.e. equals to the sum of combinatorial coefficients of the vertices $A$ in the set $V\left(A_{1}, \ldots, A_{n-1}\right)$. The theorem is proven.

Remark. Theorem 2 is useful only for consistent sets of vertices $A_{1}, \ldots, A_{n-1}$ (for which there exists a common covector $\xi$ such that the maximum of scalar product with $\xi$ on the polyhedron $\Delta_{i}$ is attained precisely at the vertex $\left.A_{i}\right)$. For inconsistent sets of vertices, the set $V\left(A_{1}, \ldots, A_{n-1}\right)$ is empty.

\section{$\S 2$. Partitions and their consistencies}

In this paragraph we discuss the notion of a consistent partition of several polyhedra.

A partition of a polyhedron $\Delta$ is a finite set of polyhedra $\mathcal{R}(\Delta)$ such that

1) the union of all the polyhedra in $\mathcal{R}(\Delta)$ is equal to the polyhedron $\Delta$,

2 ) the set $\mathcal{R}(\Delta)$ contains all the faces of any polyhedron from $\mathcal{R}(\Delta)$,

3 ) the intersection of any two polyhedra in $\mathcal{R}(\Delta)$ is either empty or is a face of

these polyhedra.

The simplest example of a partition is the tautological partition of the polyhedron, which contains the polyhedron and all of its faces.

We will deal with partitions of several polyhedra simultaneously and we will consider collections containing one polyhedron from each partition. Some of such collections we will call consistent. The method of choice of consistent collections will be governed by certain conditions which we are now going to define.

Let $\mathcal{R}\left(\Delta_{1}\right), \ldots, \mathcal{R}\left(\Delta_{k}\right)$ be a collection of partitions of several polyhedra, and let $S$ be a subset in Cartesian product of these partitions. To each point $s \in S$ corresponds a collection $\Gamma_{1}(s), \ldots \Gamma_{k}(s)$ of polyhedra, where $\Gamma_{i}(s) \in \mathcal{R}\left(\Delta_{i}\right)$. Let

$$
\Gamma(s)=\Gamma_{1}(s)+\cdots+\Gamma_{k}(s)
$$

be the sum of the polyhedra in the collection, let $G$ be a face of the polyhedron $\Gamma(s)$, and let

$$
G=G_{1}+\cdots+G_{k}
$$

be the decomposition of the face $G$ into the sum of faces of the polyhedra $\Gamma_{i}(s)$.

We will say that a set $S$ possesses the property of being natural if for every point $s \in S$ and every face $G$ of the polyhedron $\Gamma(s)$ there exists a point $s_{1} \in S$ for which $\Gamma_{i}\left(s_{1}\right)=G_{i}$ and $\Gamma\left(s_{1}\right)=G$.

We will say that a set $S$ possesses the property of being additive if the polyhedra $\Gamma(s)$ are distinct for different points $s$ and if the polyhedra $\Gamma(s)$ for $s \in S$ give a partition $\mathcal{R}(\Delta)$ of the polyhedron $\Delta=\Delta_{1}+\cdots+\Delta_{k}$.

We will call a set $S$ a consistency of the partitions $\mathcal{R}\left(\Delta_{1}\right), \ldots, \mathcal{R}\left(\Delta_{k}\right)$ if it has the property of being additive and the property of being natural. To every point $s$ in the consistency $S$ corresponds a polyhedron $\Gamma(s)$ in the partition $\mathcal{R}(\Delta)$ of 
the polyhedron $\Delta=\Delta_{1}+\cdots+\Delta_{k}$ and corresponds a collection of polyhedra $\Gamma_{1}(s), \ldots, \Gamma_{k}(s)$ in the partitions $\mathcal{R}\left(\Delta_{1}\right), \ldots, \mathcal{R}\left(\Delta_{k}\right)$ such that the following holds:

$$
\Gamma(s)=\Gamma_{1}(s)+\cdots+\Gamma_{k}(s) .
$$

A collection of the polyhedra $\Gamma_{1}(s), \ldots, \Gamma_{k}(s)$ will be called consistent, the representation (1) will be called the $S$-decomposition of the polyhedron $\Gamma(s)$, and the polyhedron $\Gamma_{i}(s) \subset \mathcal{R}\left(\Delta_{i}\right)$ will be called the $i$-th component of the $S$-decomposition of the polyhedron $\Gamma\left(s_{i}\right)$.

Example. The tautological consistency of tautological partitions of the polyhedra $\Delta_{1}, \ldots, \Delta_{k}$. The set $S$ for this consistency is in the one-to-one correspondence with the faces of the polyhedron $\Delta=\Delta_{1}+\cdots+\Delta_{k}$. To each face $\Gamma$ of the polyhedron $\Delta$ corresponds a collection $\Gamma_{1}, \ldots, \Gamma_{k}$ of components of its decomposition.

Remark. The most known to me examples of consistencies are obtained from the so-called join partitions of the polyhedra $\Delta_{1}, \ldots, \Delta_{k}$ (see [7], in which is explained the role of consistencies in the theory of finite-additive measures on the polyhedra). The definition of the consistency in the work [7] is a little bit different from ours: that definition requires more conditions and then it is proven that to these extra conditions satisfy only the consistencies obtained from the join partitions.

In this paper for us will be very important the regular partitions of the polyhedra and their regular consistencies. Only regular partitions and their regular consistencies are encountered in the calculation of the product of roots of a system of equations in terms of the Newton polyhedra. It would be possible to restrict our attention to considering only regular partitions. Let us now turn to the definition of this class of partitions and their consistencies.

Let $\Delta$ be a polyhedron in a certain linear space. We will say that a polyhedron $\tilde{\Delta}$ that belongs to the product of this linear space and the real line $\mathbb{R}^{1}$ lies over the polyhedron $\Delta$ if the polyhedron $\Delta$ is the projection of the polyhedron $\tilde{\Delta}$ along the line $\mathbb{R}^{1}$. Let $e_{1}$ be a vector in the line $\mathbb{R}^{1}$ specifying its orientation. We will say that a point $x$ of the polyhedron $\tilde{\Delta}$ is an upper point of $\tilde{\Delta}$ if the ray $x+\lambda e_{1}$, where $\lambda \geq 0$, intersects $\tilde{\Delta}$ only at the point $x$. A face of the polyhedron $\tilde{\Delta}$ will be called an upper face if each of its points is an upper point of this polyhedron.

There is a partition of the polyhedron $\Delta$ related to the polyhedron $\tilde{\Delta}$, namely the partition consisting of projections of the upper faces of the polyhedron $\tilde{\Delta}$. A partition $\Delta$ related to some polyhedron lying over it is called regular.

A regular partition of the polyhedron $\Delta$ depends not on the polyhedron $\tilde{\Delta}$ itself, but only on its upper points. The set of upper points of the polyhedron $\tilde{\Delta}$ can be considered as a graph of a piecewise-linear convex function on the polyhedron $\Delta$. Conversely, to each piecewise-linear convex function on the polyhedron is related its regular partition - in the capacity of the polyhedron $\tilde{\Delta}$ we can take the convex hull of the graph of this function. If a linear function is added to the piecewise-linear function, then the regular partition will be unchanged.

Let $\mathcal{R}\left(\Delta_{1}\right), \ldots, \mathcal{R}\left(\Delta_{k}\right)$ be a collection of regular partitions of the polyhedra $\Delta_{1}, \ldots, \Delta_{k}$. Let us choose polyhedra $\tilde{\Delta}_{1}, \ldots, \tilde{\Delta}_{k}$ that lie over the polyhedra $\Delta_{1}, \ldots, \Delta_{k}$, respectively, and generate this collection of regular partitions. The choice of the polyhedra $\tilde{\Delta}_{i}$ defines the consistency of the collection of the regular partitions. Namely, we will say that the polyhedra $\Gamma_{1}, \ldots, \Gamma_{k}$ from the partitions $\mathcal{R}\left(\Delta_{1}\right), \ldots, \mathcal{R}\left(\Delta_{k}\right)$ are consistent via the polyhedra $\tilde{\Delta}_{1}, \ldots, \tilde{\Delta}_{k}$ if the Minkowski sum $\tilde{\Gamma}_{1}+\cdots+\tilde{\Gamma}_{k}$ of 
the upper faces $\tilde{\Gamma}_{i}$ of the polyhedra $\tilde{\Delta}_{i}$ lying over them is an upper face $\tilde{\Gamma}$ of the polyhedron $\tilde{\Delta}=\tilde{\Delta}_{1}+\cdots+\tilde{\Delta}_{n}$. In this way with each upper face $\tilde{\Gamma}$ of the polyhedron $\tilde{\Delta}$ is connected a collection of consistent polyhedra $\Gamma_{1}, \ldots, \Gamma_{k}$. We obtain a definition of the consistency for which the set $S$ is in a one-to-one correspondence with the set of upper faces of the polyhedron $\tilde{\Delta}$.

Let $\Delta_{1}$ and $\Delta_{2}$ be polyhedra lying in a linear space $L$ and let $\mathcal{R}\left(\Delta_{1}\right)$ and $\mathcal{R}\left(\Delta_{2}\right)$ be their regular partitions consistent via two piecewise-linear convex functions $f_{1}$ and $f_{2}$ on these polyhedra.

The next lemma gives a necessary condition for two polyhedra $\Gamma_{1}$ and $\Gamma_{2}$ from the partitions $\mathcal{R}\left(\Delta_{1}\right)$ and $\mathcal{R}\left(\Delta_{2}\right)$ to be consistent. In its formulation we identify between themselves the tangent spaces to all the interior points of the polyhedron $\Gamma_{1}$ (of the polyhedron $\Gamma_{2}$ ) and we also identify them with a linear subspace $L_{1}$ (a subspace $L_{2}$ ) of the space $L$.

Lemma 1. If two polyhedra $\Gamma_{1}$ and $\Gamma_{2}$ from the partitions $\mathcal{R}\left(\Delta_{1}\right)$ and $\mathcal{R}\left(\Delta_{2}\right)$ are consistent via the functions $f_{1}$ and $f_{2}$, then the differentials of the restrictions of these functions on the polyhedra $\Gamma_{1}$ and $\Gamma_{2}$ are constant and coincide on the space $L_{1} \cap L_{2}$.

Proof. If two polyhedra $\Gamma_{1}$ and $\Gamma_{2}$ are consistent, then there exists a covector $\xi$ on the space $L$ such that the function $\langle\xi, x\rangle+f_{1}(x)$ is constant on the polyhedron $\Gamma_{1}$ and the function $\langle\xi, x\rangle+f_{2}(x)$ is constant on the polyhedron $\Gamma_{2}$. Differentiating, we obtain that the differential of the restriction of the function $f_{1}$ on the space $L_{1}$ is equal to the covector $(-\xi)$, and the differential of the restriction of the function $f_{2}$ on the space $L_{2}$ is also equal to $(-\xi)$. This proves the lemma.

We will say that polyhedra are affinely independent if the minimum affine subspaces containing these polyhedra are independent. In other words, the polyhedra $\Delta_{1}, \ldots, \Delta_{k}$ are affinely independent if the dimension of their Minkowski sum is equal to the sum of their dimensions:

$$
\operatorname{dim}\left(\Delta_{1}+\cdots+\Delta_{k}\right)=\operatorname{dim} \Delta_{1}+\cdots+\operatorname{dim} \Delta_{k} .
$$

The following is clear:

Lemma 2. If the polyhedra $\Delta_{1}, \ldots, \Delta_{n}$ are affinely independent in $\mathbb{R}^{n}$, then either one of these polyhedra is a point, or all of these polyhedra are segments.

The following holds:

Theorem 1. Any collection $\mathcal{R}\left(\Delta_{1}\right), \ldots, \mathcal{R}\left(\Delta_{k}\right)$ of regular partitions can be made regularly consistent in such a way that any consistent collection of polyhedra will be affinely independent.

Proof. Assume that the partition $\mathcal{R}\left(\Delta_{i}\right)$ corresponds to the function $f_{i}: \Delta_{i} \rightarrow \mathbb{R}$. We will sow that adding to the functions $f_{i}$ different sufficiently general functions, we can achieve that every consistent collection of polyhedra becomes affinely independent. We will begin with the case of two polyhedra. If the consistent polyhedra $\Gamma_{1}$ and $\Gamma_{2}$ from the partitions $\mathcal{R}\left(\Delta_{1}\right)$ and $\mathcal{R}\left(\Delta_{2}\right)$ are affinely dependent, then, as can be seen from lemma 1 , they cease to be consistent by adding an almost any linear function to the function $f_{2}$. Therefore by adding an almost any linear function to the function $f_{2}$ destroys the consistency of any pair of affinely dependent polyhedra. So the theorem is proven for the regular partitions of two polyhedra. 
The general case reduces to the one considered. Indeed, we can add the polyhedra one by one to the sum and correct with the help of a linear perturbation only the function specifying the regular partition of the polyhedron being added.

Corollary. Tautological partitions of several polyhedra in $\mathbb{R}^{n}$ can be made regularly consistent in such a way that every consistent collection will contain the affinely independent faces of these polyhedra.

Proof. Indeed, the tautological partition is clearly regular because it is assigned to any linear function on the polyhedron.

Lemma 3. Any collection of partitions $\mathcal{R}\left(\Delta_{1}\right), \ldots, \mathcal{R}\left(\Delta_{k}\right)$ can be made consistent in such a way that every consistent collection of polyhedra will be affinely independent.

Proof. Let us fix $k$ sufficiently general linear functions $f_{1}, \ldots, f_{k}$. For every consistent collection of polyhedra $\Gamma_{1}(s), \ldots, \Gamma_{k}(s)$ let us consider the regular consistency of their tautological partitions via the linear functions $f_{1}, \ldots, f_{k}$. Let us consider all consistent collections of polyhedra which can be obtained by a such regular consistency for different elements $s \in S$. The union of all such consistent collections will defined a new, finer, consistency of the partitions $\mathcal{R}\left(\Delta_{1}\right), \ldots, \mathcal{R}\left(\Delta_{k}\right)$, which satisfies the conditions of lemma 2.

\section{$\S 3$. combinatorial coefficients connected with consistent partitions}

In this paragraph the notions of a characteristic map of a collection of polyhedra, a locked collection of polyhedra, etc., are generalized to the case of partitions of collections of polyhedra equiped with a fixed consistency.

Let $\mathcal{R}\left(\Delta_{1}\right), \ldots \mathcal{R}\left(\Delta_{n}\right)$ be partitions of the polyhedra $\Delta_{1}, \ldots, \Delta_{n}$, lying in some linear space, let $S$ be their consistency, and let $\mathcal{R}(\Delta)$ be the corresponding partition of the polyhedron $\Delta=\Delta_{1}+\cdots+\Delta_{n}$. A polyhedron $\Gamma$ in the partition $\mathcal{R}(\Delta)$ will be called $S$-locked if among the components of its $S$-decomposition there is at least one point (a vertex of one of the partitions $\mathcal{R}\left(\Delta_{i}\right)$ ).

Let $K_{S}$ be the union of all the $S$-locked polyhedra $\Gamma$ in the partition $\mathcal{R}(\Delta)$ of the polyhedron $\Delta$. The map

$$
f: K_{S} \rightarrow \partial \mathbb{R}_{+}^{n}
$$

of the set $K_{S}$ into the boundary of the positive octant will be called $S$-characteristic if the component $f_{i}$ of the map $f=\left(f_{1}, \ldots, f_{n}\right)$ vanishes on and only on those polyhedra $\Gamma$ in the partition $\mathcal{R}(\Delta)$ for which the $i$-th component of the $S$-decomposition is a vertex.

Lemma 1. 1. S-characteristic maps exist.

2. For an $S$-characteristic map the preimage of the center of coordinates is precisely the set of all the vertices of the partition $\mathcal{R}(\Delta)$.

3. The set of all $S$-characteristic maps is convex.

The proof of lemma 1 almost word for word repeats the proof of lemma 1 from $\S 1$, so we will skip it.

A map $h: K_{S} \rightarrow \partial \mathbb{R}_{+}^{n}$ will be called almost $S$-characteristic if

1) the component $h_{i}$ of the map $h=\left(h_{1}, \ldots, h_{n}\right)$ vanishes on the polyhedra $\Gamma$ from

the set $K_{S}$ for which the $i$-th component of the $S$-decomposition is a point; 
2) the map $h$ maps to the center of coordinates only the vertices of the partition $\mathcal{R}(\Delta)$.

It is easy to check the following:

Lemma 2. Let $f$ be an $S$-characteristic map and let $h$ be an almost $S$-characteristic map. Then the map $h_{t}=t f+(1-t) h$ with $0<t \leq 1$ is $S$-characteristic.

In the definitions below for us will be important only the behaviour of the consistencies on the boundary of the polyhedron $\Delta$. A vertex of the partition $\mathcal{R}(\Delta)$ will be called $S$-critical if it lies on the boundary $\partial \Delta$ of the polyhedron $\Delta$ and belongs to the interior of the set $\partial \Delta \cap K_{S}$ in topology of the boundary of the polyhedron $\Delta$. Assume further that the polyhedron $\Delta$ lies in a linear space $L$ of dimension $n$; let us fix the orientations of the spaces $L$ and $\mathbb{R}^{n}$.

Definition. The combinatorial coefficient $C_{A}$ of an $S$-critical vertex $A$ of the partition $\mathcal{R}(\Delta)$ is the local degree of the germ of the map

$$
f:(\partial \Delta, A) \rightarrow\left(\partial \mathbb{R}_{+}^{n}, 0\right)
$$

of the restriction $f$ of an $S$-characteristic map on the boundary of the polyhedron.

Lemma 3. 1. The combinatorial coefficient of an $S$-critical vertex is well-defined, i.e. it does not depend on the choice of the $S$-characteristic map.

2. The local degree of the germ of restriction of an almost $S$-characteristic map on $\partial \Delta$ at an $S$-critical vertex of the partition is equal to the combinatorial coefficient of this vertex.

Proof. The first part follows by lemma 1, and the second part follows by lemma 2 .

Let us now discuss the behaviour of combinatorial coefficient when looking at finer partitions. Let $\mathcal{R}_{1}\left(\Delta_{i}\right)$ and $\mathcal{R}_{2}\left(\Delta_{i}\right), i=1, \ldots, n$ be two collections of partitions of the polyhedra $\Delta_{1}, \ldots, \Delta_{n}$ equiped with the consistencies $S_{1}$ and $S_{2}$, correspondingly. Let $\mathcal{R}_{1}$ and $\mathcal{R}_{2}$ be the corresponding partitions of the polyhedron $\Delta=\Delta_{1}+\cdots+\Delta_{k}$. We will say that the consistency $S_{2}$ of the partitions $\mathcal{R}_{2}\left(\Delta_{i}\right)$ is finer then the consistency $S_{1}$ of the partitions $\mathcal{R}_{1}\left(\Delta_{i}\right)$ if for every point $s_{2} \in S_{2}$ there exists a point $s_{1} \in S_{1}$ such that

1) the inclusion $G\left(s_{2}\right) \subseteq \Gamma\left(s_{1}\right)$ is valid, where $G\left(s_{2}\right), \Gamma\left(s_{1}\right)$ are the polyhedra corresponding to the points $s_{2} \in S_{2}$ and $s_{1} \in S_{1}$ in the partitions $\mathcal{R}_{2}(\Delta)$ and $\mathcal{R}_{1}(\Delta)$

2) for every index $1 \leq i \leq k$ the inclusion $G_{i}\left(s_{2}\right) \subseteq \Gamma_{i}\left(s_{1}\right)$ is valid, where $G_{i}\left(s_{2}\right)$, $\Gamma_{i}\left(s_{1}\right)$ are, correspondingly, the $i$-th components of the $S_{2}$ - and $S_{1}$-decompositions of the polyhedra $G\left(s_{2}\right)$ and $\Gamma\left(s_{1}\right)$.

Example. Any consistency of any partitions of any collection of polyhedra is finer than the tautological consistency of the tautological partitions of this collection of polyhedra.

Theorem 1. Let a consistency $S_{2}$ of a collection of partitions $\mathcal{R}_{2}\left(\Delta_{i}\right)$ be finer than a consistency $S_{1}$ of a collection of partitions $\mathcal{R}_{1}\left(\Delta_{i}\right)$. Suppose that an $S_{2}$ critical vertex $A \in \partial \Delta$ of the partition $\mathcal{R}_{2}(\Delta)$ belongs to a polyhedron $\Gamma$ from the partition $\mathcal{R}_{1}(\Delta)$ lying on the boundary of the polyhedron $\Delta$. Suppose that the polyhedron $\Gamma$ belongs to the interior of the set $K_{S_{1}} \cap \partial \Delta$. Then if the polyhedron $\Gamma$ has positive dimensionality, then the combinatorial coefficient $C_{A}\left(S_{2}\right)$ is equal to 
0. If, on the other hand, the polyhedron $\Gamma$ is a vertex of the partition $\mathcal{R}_{1}(\Delta)$, then $C_{A}\left(S_{2}\right)=C_{A}\left(S_{1}\right)$.

Proof. Let $f$ and $g$ be, correspondingly, an $S_{1}$-characteristic map and an $S_{2^{-}}$ characteristic map of the sets $K_{S_{1}}$ and $K_{S_{2}}$ into the boundary of the positive octant $\partial \mathbb{R}_{+}^{n}$. Consider the restrictions of the maps $f$ and $g$ on a small sphere $S^{n-1}$ surrounding the vertex $A$ in the boundary $\partial \Delta$ of the polyhedron $\Delta$. It is easy to see that under the linear homotopy $f_{t}=t f+(1-t) g$ the small sphere $S^{n-1}$ gets mapped into $\partial \mathbb{R}_{+}^{n} \backslash 0$, the complement of the center of coordinates in the boundary of the positive octant. This proves theorem 1.

We will say that a partition of a collection of $n$ polyhedra $\Delta_{1}, \ldots, \Delta_{n}$ in a linear space $L$ is $S$-developed on the boundary if the set $K_{S}$ contains the boundary of the polyhedron $\Delta$. For an $S$-developed on the boundary collection of partitions a combinatorial coefficient is defined at every vertex of the partition $\mathcal{R}(\Delta)$ which lies on the boundary of the polyhedron $\Delta$.

For $i=1, \ldots, n-1$ let us fix certain vertices $A_{i}$ in the partitions of the polyhedra $\Delta_{i}$. Consider the set $V\left(A_{1}, \ldots, A_{n-1}\right)$ of vertices of the partition of the polyhedron $\Delta$ belonging to the boundary of this polyhedron for which the points $A_{1}, \ldots, A_{n-1}$ are the components of the $S$-decomposition.

Theorem 2. For every $S$-developed on the boundary partition of a collection of polyhedra $\Delta_{1}, \ldots, \Delta_{n}$ and for every choice of vertices $A_{i}$ of the partitions $\mathcal{R}\left(\Delta_{i}\right)$ the following is true. The sum of the combinatorial coefficients of the vertices $A$ of the partition $\mathcal{R}(\Delta)$ of the polyhedron $\Delta$ belonging to the set $V\left(A_{1}, \ldots, A_{n-1}\right)$ is 0 :

$$
\sum_{A \in V\left(A_{1}, \ldots, A_{n-1}\right)} C_{A}(S)=0
$$

The proof of theorem 2 is almost word for word repeating the proof of theorem 2 from $\S 1$ and so we will skip it.

Remark. Theorem 2 is useful only for consistent collections of points $A_{1}, \ldots, A_{n-1}$ lying on the boundaries of the polyhedra $\Delta_{1}, \ldots, \Delta_{n-1}$.

Let $S$ be a consistency of a collection of partitions of the polyhedra $\Delta_{1}, \ldots, \Delta_{n}$, and let $\mathcal{R}(\Delta)$ be the corresponding partition of the polyhedron $\Delta=\Delta_{1}+\cdots+\Delta_{n}$.

We will say that a vertex $A$ of this partition is $S$-locked in the polyhedron $\Gamma \subset \mathcal{R}(\Delta)$ if $A$ is a locked vertex of the polyhedron $\Gamma$ relative to its $S$-decomposition $\Gamma=\Gamma_{1}(s)+\cdots+\Gamma_{n}(s)$. For an $S$-locked vertex $A$ in the polyhedron $\Gamma$ let us denote by $C_{A}(\Gamma, S)$ the combinatorial coefficient of this vertex in the polyhedron $\Gamma$.

Theorem 3. Suppose that a vertex $A$ of the partition $\mathcal{R}(\Delta)$ is $S$-locked in all the polyhedra $\Gamma$ from the partition $\mathcal{R}(\Delta)$ which contains this vertex. Then the sum of numbers $C_{A}(\Gamma, S)$ over all the polyhedra $\Gamma$ is equal to

1) zero, if $A$ is an interior point of the polyhedron $\Delta$;

2) the combinatorial coefficient $C_{A}(S)$, if $A$ lies in the boundary of the polyhedron

$\Delta$.

Proof. The proof of this theorem is based on a simple homological reasoning. Let $O_{n-1}$ be a $(n-1)$-dimensional skeleton of the partition $\mathcal{R}(\Delta)$, i.e. the union of all the polyhedra of the partition whose dimensionality does not exceed $n-1$. The polyhedra $\Gamma_{n-1} \subset O_{n-1}$ of the dimensionality $n-1$ can be of two types: interior 
and boundary. Every interior polyhedron $\Gamma_{n-1}$ enters in the boundary of exactly two $n$-dimensional polyhedra from the partition $\mathcal{R}(\Delta)$. Further the orientations of the polyhedron $\Gamma_{n-1}$ as the boundary of these polyhedra are different. Every boundary polyhedron $\Gamma_{n-1}$ enters in the boundary of exactly one $n$-dimensional polyhedron $\Gamma_{n}$, further its orientation as the boundary of the polyhedron $\Gamma_{n}$ is the same as the orientation of the boundary $\partial \Delta$.

The intersection of the skeleton $O_{n-1}$ with a small neighbourhood of the point $A$ is by definition contained in the set $K_{S}$. Therefore we have a well-defined germ

$$
f:\left(O_{n-1}, A\right) \rightarrow\left(\partial R_{+}^{n}, 0\right)
$$

of the restrictions of a characteristic map on the skeleton.

The degree of the restriction of the germ of the map $f$ on the boundary of the polyhedron $\Delta$ is equal to the coefficient $C_{A}(S)$. The degree of the restriction of the germ of the map $f$ on the boundary of any $n$-dimensional polyhedron $\Gamma, A \in \Gamma$, $\Gamma \subset \mathcal{R}(\Delta)$, is equal to the coefficient $C_{A}(\Gamma, S)$.

For the calculation of these degrees let us fix a small neighbourhood $U$ of the point $A$ in the $(n-1)$-dimensional skeleton $O_{n-1}$ and let us take a regular value $c \in \partial \mathbb{R}_{+}^{n}$ of the map $f$ sufficiently close to the point 0 . Consider all the preimages of point $c$ lying in the neighbourhood $U$.

The coefficient $C_{A}(\Gamma, S)$ is equal to the calculated considering the signs of such preimages of the point $c$ on the boundary $\partial \Gamma$. Each preimage of a point $c$ belonging to an interior polyhedron $\Gamma_{n-1}$ will appear twice in the sum of numbers $C_{A}(\Gamma, S)$, each time with a different sign. Each preimage of a point $c$ belonging to a boundary $\Gamma_{n-1}$ will appear only once in the sum of the numbers $C_{A}(\Gamma, S)$, moreover it will appear with the right sign. The theorem now follows.

\section{$\S 4$. A new formula for the mixed volume}

In this paragraph we formulate and proof a new formula for the mixed volume of polyhedra. In the general case this formula depends on the selection of specially consistent subpartitions of the polyhedra. However in the case most important for later on, when the collection of polyhedra is developed, the formula for the mixed volume depends only on the polyhedra themselves, and the subpartitions are not involved. In this case the formula for the mixed volume can be easily differentiated with respect to the displacements of the vertices of polyhedra. The formula for the derivative of the mixed volume by the vertex of the polyhedron is used in $\S 11$.

Let us first consider one simple but an important example. Let $I_{1}, \ldots, I_{n}$ be $n$ transversal segments in $\mathbb{R}^{n}$, and let $\Delta$ be their Minkowski sum.

Lemma 1. The volume $\mathrm{Vol} \Delta$ of the parallelepiped $\Delta=I_{1}+\cdots+I_{n}$ is given by the following formula:

$$
\operatorname{Vol} \Delta=\sum_{A \in \Delta}(-1)^{n} C_{A} \operatorname{det}\left(A_{1}, \ldots, A_{n}\right),
$$

where the summation is conducted over all the vertices $A$ of the parallelepiped $\Delta, C_{A}$ is the combinatorial coefficient at the vertex $A$, and $\operatorname{det}\left(A_{1}, \ldots, A_{n}\right)$ is the determinant of the matrix consisting of the vectors $A_{1}, \ldots, A_{n}$ which are the components of the decomposition of the vertex $A$. 
Proof. For every segment $I_{i}$ let us fix one of its vertices $l_{i}$ which we will call left, the second vertex $h_{i}$ of this segment, which will be called right, is of the form $h_{i}=l_{i}+a_{i}$, where $a_{i}$ is the vector from the first vertex of the segment to the second. Consider the function $\varphi$ assigning to $n$ vectors $x_{1}, \ldots, x_{n}$ of the space $\mathbb{R}^{n}$ the determinant $\operatorname{det}\left(x_{1}, \ldots, x_{n}\right)$ of the matrix consisting of these vectors. This function is multilinear. Using the calculation of combinatorial coefficients of the vertices of the parallelepiped $\Delta$ from theorem 1 in $\S 1$, we obtain

$$
\operatorname{det}\left(a_{1}, \ldots, a_{n}\right)=\operatorname{sign} \operatorname{det}\left(a_{1}, \ldots, a_{n}\right) \sum_{A \in \Delta}(-1)^{n} C_{A} \operatorname{det}\left(A_{1}, \ldots, A_{n}\right) .
$$

This is exactly the required statement since

$$
\operatorname{Vol} \Delta=\operatorname{sign} \operatorname{det}\left(a_{1}, \ldots, a_{n}\right) \operatorname{det}\left(a_{1}, \ldots, a_{n}\right) .
$$

We will say that a consistency $S$ of partitions $\mathcal{R}\left(\Delta_{1}\right), \ldots, \mathcal{R}\left(\Delta_{k}\right)$ has the property of inheritance if for every subset $I=\left(i_{1}, \ldots, i_{m}\right)$ of the segment of integers $(1, \ldots, k)$ the image $S_{I}$ of the set $S$ under the projection $\prod_{1 \leq i \leq k} \mathcal{R}\left(\Delta_{i}\right) \rightarrow \prod_{i \in I} \mathcal{R}\left(\Delta_{i}\right)$ defines a consistency of the partitions $\mathcal{R}\left(\Delta_{i_{1}}\right), \ldots, \mathcal{R}\left(\Delta_{i_{m}}\right)$ of the polyhedra $\Delta_{i_{1}}, \ldots, \Delta_{i_{m}}$.

It is easy to see that regular consistencies of regular partitions possess the property of inheritance. It is easy to see as well that consistent partitions of polyhedra connected to the partitions of their joins (see [7]) also possess the property of inheritance.

In a $n$-dimensional space $L$ let us fix a volume form and let us fix an orientation. Let us denote by $\operatorname{Vol}\left(\Delta_{1}, \ldots, \Delta_{n}\right)$ the mixed volume of the polyhedra $\Delta_{1}, \ldots, \Delta_{n}$ lying in the space $L$.

Lemma 2. For every collection of partitions $\mathcal{R}\left(\Delta_{i}\right)$ of the polyhedra $\Delta_{1}, \ldots, \Delta_{n}$ and for every consistency $S$ of these partitions satisfying the condition of inheritance, holds the equality

$$
\operatorname{Vol}\left(\Delta_{1}, \ldots, \Delta_{n}\right)=\sum_{s \in S} \operatorname{Vol}\left(\Gamma_{1}(s), \ldots, \Gamma_{n}(s)\right) .
$$

Proof. Since, by definition, the mixed volume is the polarization of the volume form Vol, the following equality holds:

$$
n ! \operatorname{Vol}\left(\Delta_{1}, \ldots, \Delta_{n}\right)=\sum_{I}(-1)^{n-\#|I|} \operatorname{Vol}\left(\sum_{i \in I} \Delta_{i}\right),
$$

where the outer summation is conducted over all the subsets $I$ of the segment of integers $(1, \ldots, n)$ and $\#|I|$ denotes the number of elements in the set $I$. Similarly, for every consistent collection of polyhedra we have

$$
n ! \operatorname{Vol}\left(\Gamma_{1}(s), \ldots, \Gamma_{n}(s)\right)=\sum_{I}(-1)^{n-\#|I|} \operatorname{Vol}\left(\sum_{i \in I} \Gamma_{i}(s)\right) .
$$

All that is left to finish the proof is to use the relations

$$
\operatorname{Vol}\left(\sum_{i \in I} \Delta_{i}\right)=\sum_{s \in S} \operatorname{Vol}\left(\sum_{i \in I} \Gamma_{i}(s)\right)
$$

which follow from the additivity of volume and the property of inheritance of the partition $S$. 
Lemma 3. Let $S_{1}$ be a consistency of the collection of partitions $\mathcal{R}\left(\Delta_{1}\right), \ldots, \mathcal{R}\left(\Delta_{k}\right)$ of the polyhedra $\Delta_{1}, \ldots, \Delta_{k}$ such that $S_{1}$ possesses the property of inheritance. Then it is possible to find a finer consistency $S_{2}$ of the same partitions, also possessing the property of inheritance, for which every $S_{2}$-consistent collection of polyhedra is affinely-independent.

Proof. The consistency described in the proof of lemma 3 in $\S 2$ has the required properties.

Theorem 1. For every $S$-developed on the boundary collection of partitions $\mathcal{R}\left(\Delta_{1}\right)$, $\ldots, \mathcal{R}\left(\Delta_{n}\right)$ of the polyhedra $\Delta_{1}, \ldots, \Delta_{n}$ for which the consistency $S$ possesses the property of inheritance, the following formula holds:

$$
n ! \operatorname{Vol}\left(\Delta_{1}, \ldots, \Delta_{n}\right)=(-1)^{n} \sum_{A \in \partial \Delta} C_{A}(S) \operatorname{det}\left(A_{1}, \ldots, A_{n}\right) .
$$

The summation here is conducted over all the vertices $A$ of the partition $\mathcal{R}(\Delta)$ lying on the boundary of the polyhedron $\Delta ; A_{1}, \ldots, A_{n}$ is a collection of $S$-consistent vertices in the partitions of the polyhedra $\Delta_{1}, \ldots, \Delta_{n}$ such that $A_{1}+\cdots+A_{n}=A$.

Proof. 1. Let us choose a finer consistency $S_{2}$ of the partitions $\mathcal{R}\left(\Delta_{1}\right), \ldots, \mathcal{R}\left(\Delta_{n}\right)$ which has the property of inheritance and for which every $S_{2}$-consistent collection of polyhedra is affinely-independent (see lemma 3 ). Let us denote by $\mathcal{R}_{2}(\Delta)$ the partition of the polyhedron $\Delta$ corresponding to the consistency $S_{2}$.

According to lemma 2 , the mixed volume of the polyhedra $\Delta_{1}, \ldots, \Delta_{n}$ is equal to the sum of the mixed volumes $\operatorname{Vol}\left(\Gamma_{1}(s), \ldots, \Gamma_{n}(s)\right)$ of all the $S_{2}$-consistent collections of polyhedra.

$$
\operatorname{Vol}\left(\Delta_{1}, \ldots, \Delta_{n}\right)=\sum_{s \in S_{2}} \operatorname{Vol}\left(\Gamma_{1}(s), \ldots, \Gamma_{n}(s)\right) .
$$

2. By hypothesis all $S_{2}$-consistent collections of polyhedra $\Gamma_{1}(s), \ldots, \Gamma_{n}(s)$ are affinely-independent, i.e. they satisfy the relation $\operatorname{dim}\left(\Gamma_{1}(s)+\cdots+\Gamma_{n}(s)\right)=$ $\operatorname{dim} \Gamma_{1}(s)+\cdots+\operatorname{dim} \Gamma_{n}(s)$. Therefore, either all the polyhedra $\Gamma_{i}(s)$ are segments lying on affinely-independent lines, or among the polyhedra $\Gamma_{i}(s)$ there is a point.

3. Suppose that for $s \in S_{2}$ there is a point among the polyhedra $\Gamma_{i}(s)$. Then the mixed volume $\operatorname{Vol}\left(\Gamma_{1}(s), \ldots, \Gamma_{n}(s)\right)$ is equal to zero. Furthermore, in this case the combinatorial coefficient $C_{A}\left(\Gamma, S_{2}\right)$ of every vertex $A$ of the polyhedron $\Gamma(s)=\Gamma_{1}(s)+\cdots+\Gamma_{n}(s)$ is equal to zero.

4. Suppose that for $s \in S_{2}$ all the polyhedra $\Gamma_{1}(s), \ldots, \Gamma_{n}(s)$ are affinelyindependent segments. Then the mixed volume $\operatorname{Vol}\left(\Gamma_{1}(s), \ldots, \Gamma_{n}(s)\right)$ multiplied by $n$ ! is equal to the volume of the parallelepiped $\Gamma(s)=\Gamma_{1}(s)+\cdots+\Gamma_{n}(s)$. According to lemma 1 we have

$$
\operatorname{Vol}(\Gamma(s))=\sum_{A \in \Gamma(s)}(-1)^{n} C_{A}\left(\Gamma, S_{2}\right) \operatorname{det}\left(A_{1}, \ldots, A_{n}\right)
$$

where the summation is conducted over the set of vertices $A$ in $\Gamma(s)$, and $A_{1}+\cdots+$ $A_{n}=A$. 
5. By summing up the volumes of all the parallelepipeds $\Gamma(s), s \subset S_{2}$ we obtain the relation

$$
n ! \operatorname{Vol}\left(\Delta_{1}, \ldots, \Delta_{n}\right)=(-1)^{n} \sum_{A \in \mathcal{R}_{2}(\Delta)} \operatorname{det}\left(A_{1}, \ldots A_{n}\right) \sum_{A \in \Gamma \subset \mathcal{R}_{2}(\Delta)} C_{A}\left(\Gamma, S_{2}\right) .
$$

Here the outer summation is conducted over the set of vertices $A$ of the partition $\mathcal{R}_{2}(\Delta) ; A_{1}, \ldots, A_{n}$ is the $S_{2}$-consistent collection of vertices such that $A_{1}+\cdots+$ $A_{n}=A$. The inner summation is conducted over all the polyhedra $\Gamma$ of the partition $\mathcal{R}_{2}(\Delta)$ which contain the vertex $A$.

6. According to theorem 3 from $\S 3$ applied to the consistency $S_{2}$ of the partitions $\mathcal{R}\left(\Delta_{i}\right)$, we have:

a) for every vertex $A$ of the partition $\mathcal{R}_{2}(\Delta)$ lying inside the polyhedron $\Delta$, the sum

$$
\sum_{A \in \Gamma \subset \mathcal{R}_{2}(\Delta)} C_{A}\left(\Gamma, S_{2}\right)
$$

is equal to zero;

b) for every boundary vertex $A \in \partial \Delta$ of the partition $\mathcal{R}_{2}(\Delta)$, the sum

$$
\sum_{A \in \Gamma \subset \mathcal{R}_{2}(\Delta)} C_{A}\left(\Gamma, S_{2}\right)
$$

is equal to $C_{A}\left(S_{2}\right)$.

7. According to theorem 1 from $\S 2$ we have $C_{A}\left(S_{2}\right)=0$ for every point $A$ which is not a vertex of the partition $\mathcal{R}(\Delta)$, and $C_{A}\left(S_{2}\right)=C_{A}(S)$ for every point $A$ which is a vertex of the partition $\mathcal{R}(\Delta)$.

8. By substituting in the formula in item 5 the expressions for the sums of combinatorial coefficients from items 6 and 7 , we obtain the desired formula. Theorem 1 is proven.

Let us discuss the new formula for the mixed volume from theorem 1.

Lemma 4. For every $S$-developed on the boundary collection of partitions $\mathcal{R}\left(\Delta_{1}\right), \ldots, \mathcal{R}\left(\Delta_{n}\right)$ of the polyhedra $\Delta_{1}, \ldots, \Delta_{n}$, the value

$$
n ! V_{S}\left(\Delta_{1}, \ldots, \Delta_{n}\right)=(-1)^{n} \sum_{A \in \partial \Delta} C_{A}(S) \operatorname{det}\left(A_{1}, \ldots, A_{n}\right)
$$

is conserved under parallel translations of the polyhedra $\Delta_{1}, \ldots, \Delta_{n}$.

Proof. Let us show, for example, then the value $n ! V_{S}\left(\Delta_{1}, \ldots, \Delta_{n}\right)$ is conserved under the parallel translation of the polyhedron $\Delta_{n}$ by the vector $a$. Indeed,

$$
\begin{array}{r}
n ! V_{S}\left(\Delta_{1}, \ldots, \Delta_{n-1}, \Delta_{n}+a\right)-n ! V_{S}\left(\Delta_{1}, \ldots, \Delta_{n}\right)= \\
=(-1)^{n} \sum_{A \in \partial \Delta} C_{A}(S) \operatorname{det}\left(A_{1}, \ldots, A_{n-1}, a\right)= \\
=(-1)^{n} \sum_{A_{1}, \ldots, A_{n-1}} C_{A}(S) \operatorname{det}\left(A_{1}, \ldots, A_{n-1}, a\right) \sum_{A \in \partial \Delta} C_{A}(S),
\end{array}
$$


where the inner summation is conducted over all the vertices $A$ of the partition $\mathcal{R}(\Delta)$ for which the points $A_{i}$ for $1 \leq i<n$ are the $i$-th components of its $S$ decomposition. By virtue of theorem 2 in $\S 3$ all the inner sums $\sum_{A \in \partial \Delta} C_{A}(S)$ are equal to 0 . The lemma is proven.

Remark. For consistencies $S$ with the property of inheritance (which holds for all main examples of consistencies), lemma 4 follows from theorem 1 . Theorem 2 in $\S 3$ and theorem 2 in $\S 1$ were found after theorem 1 . The point of depart was the fact that the mixed volume is conserved under parallel translations of the polyhedra.

Theorem 2. For every developed collection of the polyhedra $\Delta_{1}, \ldots, \Delta_{n}$ the identity

$$
n ! \operatorname{Vol}\left(\Delta_{1}, \ldots, \Delta_{n}\right)=(-1)^{n} \sum C_{A} \operatorname{det}\left(A_{1}, \ldots, A_{n}\right) .
$$

holds. Here the summation is conducted over all the vertices $A$ of the polyhedron $\Delta$, and $A_{1}, \ldots, A_{N}$ are the components of the decomposition of the vertex $A$.

Proof. This theorem is a particular case of theorem 1 for tautological consistencies of the tautological partitions of the polyhedra $\Delta_{1}, \ldots, \Delta_{n}$.

Remark. Theorem 2 was initially found for the case when the polyhedra $\Delta_{1}, \ldots, \Delta_{n}$ have integral vertices [4]. The proof in [4] is based in algebraic geometry and it cannot be extended to the case of non-integral polyhedra. With certain extra assumptions, theorem 2 was proven in [5]. These extra assumptions are crucial for the proof and the proof in [5] cannot be carried over to the general case.

Let $\Delta$ be a polyhedron in $\mathbb{R}^{n}$, let $A$ be one of its vertices, let $L$ be the set of the other vertices of this polyhedron $\Delta$. For every vector $h$ we can consider the point $(A+h)$ and define the polyhedron $\Delta(A+h)$ as the convex hull of the set $L \cup(A+h)$. For example, by definition, the polyhedron $\Delta(A+0)$ coincides with the polyhedron $\Delta$.

Now let $\Delta_{1}, \ldots, \Delta_{n}$ be a collection of polyhedra in $\mathbb{R}^{n}$, and let $A_{i}$ be one of the vertices of the polyhedron $\Delta_{i}$. Let us consider the mixed volume $V(h)$ of the polyhedra $\Delta_{1}, \ldots, \Delta_{i-1}, \Delta_{i}(A+h), \Delta_{i+1}, \ldots, \Delta_{n}$ as a function of the vector $h$.

Definition. Suppose that the function $V(h)$ is differentiable by $h$ at the point 0 , and let $d V$ be its differential. We will call this differential the derivative of the mixed volume by the vertex $A$ of the polyhedron $\Delta_{i}$ and we will denote this differential by the symbol $d_{A_{i}} \operatorname{Vol}\left(\Delta_{1}, \ldots, \Delta_{n}\right)$.

Theorem 3. The mixed volume of a developed collection of the polyhedra $\Delta_{1}, \ldots, \Delta_{n}$ is differentiable with respect to every vertex of every polyhedron. The value of the derivative $d_{A_{i}} \operatorname{Vol}\left(\Delta_{1}, \ldots, \Delta_{n}\right)$ of the mixed volume by the vertex $A_{i}$ of the polyhedron $\Delta_{i}$ on the vector $k \in \mathbb{R}^{n}$ is given by the following formula:

$$
d_{A_{i}} \operatorname{Vol}\left(\Delta_{1}, \ldots, \Delta_{n}\right)(k)=\frac{1}{n !} \sum_{A}(-1)^{n} C_{A} \operatorname{det}\left(A_{1}, \ldots, A_{i-1}, k, A_{i+1}, \ldots, A_{n}\right),
$$

where the summation is conducted over vertices $A$ of the polyhedron $\Delta=\Delta_{1}+$ $\cdots+\Delta_{n}$ for which the $i$-th component of the decomposition coincides with the vertex $A_{i}$, and $A_{j}$ for $j \neq i$ denotes the $j$-th component of the decomposition of the vertex $A$.

Proof. Theorem 3 follows right away from the formula in theorem 2 for the mixed volumes of a developed collection of polyhedra. 


\section{$\S 5$. Product in a torus of points which depend algebraically on parameters}

In this paragraph we turn to algebraic geometry and make general remarks about product of points, which algebraically depend on a parameter, in the group $\left(\mathbb{C}^{*}\right)^{n}$. Theorem 3 in this paragraph plays an important role in further calculations.

Let $f$ and $g$ be two meromorphic functions on a compact algebraic curve. Let us define the function $R(f, g)$ of one complex variable $t$ by the following formula:

$$
R(f, g)(t)=\prod_{f(a)=t} g(a) .
$$

In this formula the product is taken over all the preimages $a$ of the point $t$ of the map $f, f(a)=t$; the number $g(a)$ enters in the product the number of times equal to the multiplicity of the root $a$ of the equation $f(a)=t$. The function $R(f, g)$ is a single-valued algebraic function, therefore it is a rational function. The main term of the asymptotic of the function $R(f, g)$ with $t \rightarrow \infty$ can be defined by the main terms of the asymptotics of the functions $f$ and $g$ at the poles of the function $f$.

Theorem 1. Let $P$ be the set of poles of the function $f$ and suppose that at the points $a$ in $P$ we are given the main terms of the expansions of the functions $f$ and g. Namely, suppose that

$$
f=c_{1}(a) u^{-k_{1}(a)}+\ldots, \quad g=c_{2}(a) u^{-k_{2}(a)}+\ldots,
$$

where $u$ is a local parameter on the curve in the neighbourhood of the point $a$, $u(a)=0$. Then the main term of the asymptotic of the function $R(f, g)$ with $t \rightarrow \infty$ can be computed using these data. Namely, $R(f, g)=c t^{k}+\ldots$, where $k=\sum_{a \in P} k_{2}(a)$, and

$$
c=\prod_{a \in P} c_{1}^{-k_{2}(a)}(a) c_{2}^{k_{1}(a)}(a)(-1)^{k_{2}(a)+k_{1}(a) k_{2}(a)} .
$$

Proof. In the neighbourhood of a pole $a$ if the function $f$ we have $f=c_{1}(a) u^{-k_{1}(a)}+$ $\ldots, g=c_{2}(a) u^{-k_{2}(a)}+\ldots$ For large $t$ the equation $f=t$ has $k_{1}(a)$ solutions near the point $a$ with the asymptotic $u=t^{-\frac{1}{k_{1}(a)}} c_{1}(a)^{\frac{1}{k_{1}(a)}} \varepsilon+\ldots$, where $\varepsilon$ is a root of unity of degree $k_{1}(a)$. On these solutions

$$
g \sim t^{\frac{k_{2}(a)}{k_{1}(a)}} c_{1}(a)^{-\frac{k_{2}(a)}{k_{1}(a)}} c_{2}(a) \varepsilon^{k_{2}(a)}+\ldots
$$

The product of the values of the function $g$ over all the solutions of the equation $f=t$ lying in a neighbourhood of a point $a$ is of the form

$$
\begin{aligned}
\prod t^{\frac{k_{2}(a)}{k_{1}(a)}} c_{1}(a)^{-\frac{k_{2}(a)}{k_{1}(a)}} c_{2}(a) \varepsilon^{k_{2}(a)}+\cdots= & \\
& t^{k_{2}(a)} c_{1}^{-k_{2}(a)} c_{2}^{k_{1}(a)}(-1)^{\left(k_{1}(a)+1\right) k_{2}(a)}+\ldots
\end{aligned}
$$

(According to Vieta formula, the product of all the roots of unity of degree $k_{1}(a)$ is equal to $(-1)^{k_{1}(a)+1}$; here we used this fact.) The main term of the asymptotic of the function $R(f, g)(t)$ is equal to the product of the found main terms of the asymptotics over all the poles of the function $f$. Theorem 1 now follows. 
Theorem 2. Suppose that $f$ and $g$ are two meromorphic functions on a compact algebraic curve. Suppose further that the support of the divisor of the function $f$ contains the support of the divisor of the function $g$ (i.e. if at some point the function $g$ is 0 or $\infty$, then the function $f$ at this point is also either 0 or $\infty$ ). Then the equality $R(f, g)(t)=c t^{k}$ is true. The constant $c$ and the degree $k$ appearing in this equality can be computed explicitly by the main terms of the functions $f$ and $g$ at the poles of the function $f$.

Proof. According to theorem 2 the function $R(f, g)$ has neither zeros nor poles in $\mathbb{C}^{*}$. Indeed, if the function $f$ at a point $a$ is equal to $t$ and $t \neq 0, t \neq \infty$, then by hypothesis the function $g(a)$ is neither zero nor infinity as well. A rational function having no zeros nor poles in $\mathbb{C}^{*}$ is clearly equal to $c t^{k}$. To determine the constants $c$ and $k$ it is enough to use the computation in theorem 1 of the asymptotic of this function as $t \rightarrow \infty$.

Suppose that $\pi: \bar{X} \rightarrow\left(\mathbb{C}^{*}\right)^{n}$ is a meromorphic map of a compact curve $\bar{X}$ in the complex torus $\left(\mathbb{C}^{*}\right)^{n}$ with coordinates $x_{1}, \ldots, x_{n}$. This map is given by a collection of meromorphic functions $f_{i}=\pi^{*} x_{i}$. Let us begin with the definition of the asymptotic power lines for the curve $X=\pi(\bar{X})$ in the torus $\left(\mathbb{C}^{*}\right)^{n}$. Let $D$ be the union of the supports of the divisors of the functions $f_{1}, \ldots, f_{n}$. In the neighbourhood of a point $a \in D$ let us choose a local parameter $u$ such that $u(a)=0$. Suppose that $c_{1}(a) u^{-k_{1}(a)}, \ldots, c_{n}(a) u^{-k_{n}(a)}$ are the main terms of the Laurent series for the functions $f_{1}, \ldots, f_{n}$ in the neighbourhood of the point $a$. The curve

$$
x_{1}=c_{1}(a) u^{-k_{1}(a)}, \ldots, x_{n}=c_{n}(a) u^{-k_{n}(a)}
$$

will be called the asymptotic power line of the curve $X$ corresponding to the point $a \in D$. The curve (2) depends on the point $a$ but is independent of the choice of the local parameter $u$.

In future we will deal with curves in the torus $\left(\mathbb{C}^{*}\right)^{n+1}$ having one selected coordinate $\left(\mathbb{C}^{*}\right)^{n+1}=\left(\mathbb{C}^{*}\right)^{n} \times \mathbb{C}^{*}$. The asymptotic line

$$
x_{1}=c_{1}(a) u^{-k_{1}(a)}, \ldots, x_{n+1}(a)=c_{n+1}(a) u^{-k_{n+1}(a)}
$$

of the curve $X$ will be called horizontal if the degree $k_{n+1}(a)$ is equal to 0 . We will say that along an asymptotic line the last coordinate approaches $\infty$ if the degree $k_{n+1}(a)$ is positive.

Theorem 3. Suppose that $X_{1}$ and $X_{2}$ are two curves in $\left(\mathbb{C}^{*}\right)^{n} \times \mathbb{C}^{*}$. Suppose further that these curves do not have horizontal asymptotes and have exactly the same collection of asymptotic power lines on which the last coordinate approaches infinity. Then for every value $t_{0}$ of the parameter $t$ the product in the group $\left(\mathbb{C}^{*}\right)^{n} \times \mathbb{C}^{*}$ of all the points of intersection of the first curve $X_{1}$ with the hyperplane $t=t_{0}$ and the product of all the points of intersection of the second curve $X_{2}$ with the same hyperplane $t=t_{0}$ are equal.

The proof is obtained by applying theorem 2 to the normalizations $\bar{X}_{1}$ and $\bar{X}_{2}$ of the curves $X_{1}$ and $X_{2}$. In the capacity of the function $f$ we choose the coordinate $t$ and in the capacity of the function $g$ we choose one by one all the other coordinates in the torus $\left(\mathbb{C}^{*}\right)^{n}$. Since the curve $X_{1}$ does not have horizontal asymptotes, none 
of the coordinates functions $x_{1}, \ldots, x_{n}$ can become zero or infinity when $t \neq 0$ and $t \neq \infty$. Therefore theorem 2 is applicable and each of the coordinate functions of the product of the points of intersection of the curve $X_{1}$ with the hyperplane $t$ is the monomial $c t^{k}$. The same is true for the curve $X_{2}$. Moreover, the leading terms of the asymptotic of the coordinate functions at the poles of the function $t$ on the curves $X_{1}$ and $X_{2}$ are exactly the same, because the curves $X_{1}$ and $X_{2}$ have the same asymptotic power lines on which the last coordinate $t$ approaches $\infty$. Therefore theorem 3 follows from theorem 2 .

Let $H$ be an algebraic hypersurface in $\mathbb{C}^{N}$, let $H=H_{1} \cup \cdots \cup H_{k}$ be the decomposition of $H$ into irreducible components, and let $R_{1}, \ldots, R_{k}$ be the polynomials in $\mathbb{C}^{N}$ defining the irreducible equations of these components, i.e. $R_{i}=0$ is the irreducible equation for the component $H_{i}$. The functions $R_{i}$ are defined up to multiplication by a nonzero constant.

The map $R:\left(\mathbb{C}^{N} \backslash H\right) \rightarrow\left(\mathbb{C}^{*}\right)^{k}$, where $R=R_{1}, \ldots, R_{k}$, will be called the characteristic map of the complement $\mathbb{C}^{N} \backslash H$ of the algebraic hypersurface $H$ in the space $\mathbb{C}^{N}$. The characteristic map is defined uniquely up to renumbering the components of the map $R$ and multiplying the map by a constant $c \in\left(\mathbb{C}^{*}\right)^{k}$.

Let us consider a multidimensional algebraic manifold $X$ together with its meromorphic mapping $\pi: X \rightarrow \mathbb{C}^{N}$ in the $N$-dimensional complex space and its meromorphic mapping $g: X \rightarrow\left(\mathbb{C}^{*}\right)^{n}$ in the $n$-dimensional complex torus.

Let us assume that in $\mathbb{C}^{N}$ there exist an algebraic hypersurface $H$ and an algebraic subset $\Sigma$ of codimension at least 2 such that

1 ) the manifold $X$ together with its projection $\pi$ define a ramified finite-sheeted covering over the set $U=\left(\mathbb{C}^{N} \backslash H\right) \backslash \Sigma$. This means that every point in the set $U$ has a finite number of preimages under the mapping $\pi$, and this number of preimages, counted with multiplicity, is constant and not equal to zero;

2) the meromorphic map $g: X \rightarrow\left(\mathbb{C}^{*}\right)^{n}$ is regular on the preimage $\pi^{-1}(U)$ of the set $U$.

Suppose that the conditions 1) and 2) are satisfied. Let us define the function $R(\pi, g)$ on $U$ by the following formula:

$$
R(\pi, g)(x)=\prod_{\pi(a)=x} g(a) .
$$

In this formula the product is taken over all the preimages $a$ of the point $x$ under the mapping $\pi, \pi(a)=x$, and the element $g(a)$ in the group $\left(\mathbb{C}^{*}\right)^{n}$ enters in the product (1) the number of times equal to the multiplicity of the root $a$ of the equation $\pi(a)=x$.

Theorem 4. The map $R(\pi, g): U \rightarrow\left(\mathbb{C}^{*}\right)^{n}$ is regular. Suppose that $R:\left(\mathbb{C}^{N} \backslash\right.$ $H) \rightarrow\left(\mathbb{C}^{*}\right)^{k}$ is a characteristic map of the complement of the hypersurface $H$ in the space $\mathbb{C}^{N}$. Then there exist a constant $c \in\left(\mathbb{C}^{*}\right)^{n}$ and a homomorphism $\tau:\left(\mathbb{C}^{*}\right)^{k} \rightarrow\left(\mathbb{C}^{*}\right)^{n}$ such that $R(\pi, g)=c \tau \circ R$ on the region $U$.

Proof. Every rational function with no zeros nor poles in the region $\mathbb{C}^{N} \backslash H$ is clearly of the form $c_{1} R_{1}^{m_{1}} \ldots R_{k}^{m_{k}}$, where $c_{1} \neq 0$ and $m_{i}$ is an integer number. Furthermore, every component $R_{i}(\pi, g)$ of the product $R(\pi, g)$ is a rational function in the region $U$ with no zeros nor poles in this region. By hypothesis the set $\Sigma$ has codimension at least 2 , therefore the function $R_{i}$ is regular in the set $\mathbb{C}^{N} \backslash H$ as well, moreover has no zeros nor poles in this region. The theorem follows. 


\section{$\S 6$. Product of roots of a system of equations}

In this paragraph we consider the problem of calculating the product of the roots of a sufficiently general system of equations with fixed Newton polyhedra. We show that without making any assumptions about the choice of the Newton polyhedra, this problem is just as difficult as the problem of finding the multidimensional resultants (see, for example, [3]). Suppose that

$$
P_{1}=\cdots=P_{n}=0
$$

is a system of equations in $\left(\mathbb{C}^{*}\right)^{n}$, where $P_{1}, \ldots, P_{n}$ are Laurent polynomials whose supports lie, correspondingly, in the Newton polyhedra $\Delta_{1}, \ldots, \Delta_{n}$.

It is known [1] that almost all the systems (1) have exactly the same number of solutions equal to $n ! \operatorname{Vol}\left(\Delta_{1}, \ldots, \Delta_{n}\right)$, where $\operatorname{Vol}\left(\Delta_{1}, \ldots, \Delta_{n}\right)$ is the mixed volume of the polyhedra $\Delta_{1}, \ldots, \Delta_{n}$. Let us denote by $M\left(P_{1}, \ldots, P_{n}\right)$ the point in the torus $\left(\mathbb{C}^{*}\right)^{n}$ which is equal to the product of roots of the system (1). By doing this, we assume that the number of roots of the system (1), counted with multiplicity, is equal to $n ! \operatorname{Vol}\left(\Delta_{1}, \ldots, \Delta_{n}\right)$, and that every roots of the equation enters in the product the same number of times as its multiplicity.

Remark. The function $M\left(P_{1}, \ldots, P_{n}\right)$, assigning to the sufficiently general Laurent polynomials $P_{1}, \ldots, P_{n}$ the product of the roots in $\left(\mathbb{C}^{*}\right)^{n}$ of the system of equations (1), by definition depends on the Newton polyhedra $\Delta_{1}, \ldots, \Delta_{n}$, because these polyhedra enter in the definition of a sufficiently general system. So, when we talk about the function $M\left(P_{1}, \ldots, P_{n}\right)$ we always mean that some collection of polyhedra $\Delta_{1}, \ldots, \Delta_{n}$ is already fixed.

Our problem is to compute the function $M\left(P_{1}, \ldots, P_{n}\right)$. We are going to describe the full solution of this problem in the case when the collection of Newton polyhedra $\Delta_{1}, \ldots, \Delta_{n}$ is developed. But first let us consider a number of general notations, definitions and comments.

Let $P(x)=\sum a_{k} x^{k}$, where $x=x_{1}, \ldots, x_{n}, k=k_{1}, \ldots, k_{n}, x^{k}=x_{i}^{k_{1}} \ldots x_{n}^{k_{n}}$, be a Laurent polynomial. With each integral polyhedron $\Gamma \subset \mathbb{R}^{n}$ and a Laurent polynomial $P$ let us associate a Laurent polynomial $P(\Gamma)$ defined by the formula $P(\Gamma)(x)=\sum a_{k}(\Gamma) x^{k}$, where $a_{k}(\Gamma)=0$ if $k \notin \Gamma$, and $a_{k}(\Gamma)=a_{k}$ if $k \in \Gamma$.

Let us consider the system of equations $P_{1}=\cdots=P_{n}=0$ in which the Laurent polynomial $P_{i}$ have support inside the fixed polyhedra $\Delta_{i}, P_{i}(x)=\sum a_{i k} x^{k}$ where $k \in \Delta$ (we do not assume here that the Newton polyhedron of the Laurent polynomial $P_{i}$ is equal to $\Delta_{i}$ - the coefficients $a_{i k}$ can be zero). To each face $\Gamma$ of the polyhedron $\Delta=\Delta_{1}+\cdots+\Delta_{n}$ corresponds the collection of faces $\Gamma_{i} \subset \Delta_{i}$ such that $\Gamma_{1}+\cdots+\Gamma_{n}=\Gamma$. Therefore with every face $\Gamma$ of the polyhedron $\Delta$ is connected a "truncated system" of equations

$$
P_{1}\left(\Gamma_{1}\right)=\cdots=P_{n}\left(\Gamma_{n}\right)=0
$$

in $\left(\mathbb{C}^{*}\right)^{n}$. If $\Gamma$ is a characteristic face of the polyhedron $\Delta$, then the system $(\Gamma)$ is, generally speaking, inconsistent. The system (1) is called $\Delta$-nondegenerate at infinity (relative to the fixed collection of polyhedra $\Delta_{1}, \ldots, \Delta_{n}$ ) if the system $(\Gamma$ ) is consistent for all proper faces $\Gamma$ of the polyhedron $\Delta$. According to Bernshtein's theorem [1] the number of roots of the system (1) is equal to $n ! \operatorname{Vol}\left(\Delta_{1}, \ldots, \Delta_{n}\right)$ if and only if the system $(1)$ is $\Delta$-nondegenerate at infinity. 
The space of all the systems of equations (1) with fixed polyhedra $\Delta_{1}, \ldots, \Delta_{n}$ can be considered as the space $\mathbb{C}^{N}=\mathbb{C}^{\left|\Delta_{1}\right|} \times \cdots \times \mathbb{C}^{\left|\Delta_{n}\right|}$, where $\left|\Delta_{i}\right|$ is the number of integral points in the polyhedron $\Delta_{i}$. To do this we have to assign to a system (1) a point in $\mathbb{C}^{N}$ whose collection of coordinates is the collection of all the coefficients of all the Laurent polynomials $P_{i}$, this assignment is performed in such a way that the coefficient $a_{i k}$ of the Laurent polynomial $P_{i}$ is equal to the coordinate in the space $\mathbb{C}^{\left|\Delta_{i}\right|}$ of the corresponding point $k \in \Delta_{i}$.

Lemma 1. The closure $B_{\Gamma}$, in the space $\mathbb{C}^{N}$ of all the systems, of the set for which the condition $(\Gamma)$ is not satisfied is an irreducible algebraic manifold.

Proof. Dividing, if necessary, every equation of the system $(\Gamma)$ on a certain monomial, we can assume that every Laurent polynomial $P_{i}\left(\Gamma_{i}\right)$ has a free term. The original system $(\Gamma)$ can be thus rewritten in the form

$$
\tilde{P}_{1}\left(\Gamma_{1}\right)(x)=a_{1}, \ldots, \tilde{P}_{n}\left(\Gamma_{n}\right)(x)=a_{n}, \quad x \in\left(\mathbb{C}^{*}\right)^{n},
$$

where $a_{i}$ is the free term of the Laurent polynomial $P_{i}\left(\Gamma_{i}\right)$ and $\tilde{P}_{i}\left(\Gamma_{i}\right)=P_{i}\left(\Gamma_{i}\right)-a_{i}$. Let us denote by $B_{\Gamma}^{\prime}$ the algebraic manifold defined by the system $\left(\Gamma^{\prime}\right)$ in the space $\mathbb{C}^{N} \times\left(\mathbb{C}^{*}\right)^{n}$. The manifold $B_{\Gamma}^{\prime}$ is irreducible because it is biregularly equivalent to the manifold $\mathbb{C}^{N-n} \times\left(\mathbb{C}^{*}\right)^{n}$. The manifold $B_{\Gamma}$ is the closure of the projection of the irreducible manifold $B_{\Gamma}^{\prime}$. Therefore it is irreducible as well.

We are going to apply the results from the previous paragraph in the following situation. Let the algebraic manifold $X$ be the direct product $\left(\mathbb{C}^{*}\right)^{n} \times \mathbb{C}^{N}$ of the torus $\left(\mathbb{C}^{*}\right)^{n}$ and the space $\mathbb{C}^{N}$ of all the systems whose equations have supports in the given polyhedra $\Delta_{1}, \ldots, \Delta_{n}$. The meromorphic maps $f: X \rightarrow \mathbb{C}^{N}$ and $g: X \rightarrow\left(\mathbb{C}^{*}\right)^{n}$ are natural projections.

Let us introduce the following notation. If the manifold $B_{\Gamma}$ has dimension $(n-1)$, let us denote it by $H_{\Gamma}$ and let us define $\Sigma_{\Gamma}=\emptyset$. If on the other hand the manifold $B_{\Gamma}$ has dimension smaller than $(n-1)$, let us denote it by $\Sigma_{\Gamma}$ and let us define $H_{\Gamma}=\emptyset$. Denote by $R_{\Gamma}$ the polynomial defining the irreducible equation $R_{\Gamma}=0$ of the hypersurface $H_{\Gamma}$ (if $H_{\Gamma}=\emptyset$, then $R_{\Gamma}=c \neq 0$ ). Let $H=\bigcup_{\Gamma \subset \Delta} H_{\Gamma}$ and $\Sigma=\bigcup_{\Gamma \subset \Delta} \Sigma_{\Gamma}$, where the union is taken over all the characteristic faces $\Gamma$ of the polyhedron $\Delta=\Delta_{1}+\cdots+\Delta_{n}$. Applying theorem 4 from $\S 5$, we obtain the following theorem.

Theorem 1. The product $M\left(P_{1}, \ldots, P_{n}\right)$ of all the roots in $\left(\mathbb{C}^{*}\right)^{n}$ of a system of equations (1) gives a regular map of the region $U=\mathbb{C}^{N} \backslash H \backslash \Sigma$ in the torus $\left(\mathbb{C}^{*}\right)^{n}$. The map $M\left(P_{1}, \ldots, P_{n}\right)$ can be regularly extended to the set $\mathbb{C}^{N} \backslash H$. Every component $M_{i}\left(P_{1}, \ldots, P_{n}\right)$ of the map $M\left(P_{1}, \ldots, P_{n}\right)$ is the product of some nonzero constant $c_{i}$ with the product of some integral powers $m_{i \Gamma}$ of the functions $R_{\Gamma}$, i.e. $M_{i}\left(P_{1}, \ldots, P_{n}\right)=c_{i} \prod_{\Gamma \subset \Delta} R_{\Gamma}^{m_{i \Gamma}}$. In particular, the product $M\left(P_{1}, \ldots, P_{n}\right)$ does not depend on the coefficients $a_{i k}$ of the Laurent polynomials $P_{i}$ which correspond to the interior points $k$ of the polyhedra $\Delta_{i}$.

Theorem 1 shows that the problem of calculating the product $M\left(P_{1}, \ldots, P_{n}\right)$ of roots of a system of equations is mainly reducible to the problem of calculating the multidimensional resultants $R_{\Gamma}$. This last problem is far from easy [3]. However there is one broad class of systems for which nonetheless we have all the reasons 
to expect a simple answer. This is the class of systems for which the collection of polyhedra $\Delta_{1}, \ldots, \Delta_{n}$ is developed. In this case every resultant $R_{\Gamma}$ is either a constant or one of the coefficients of one of the Laurent polynomials $P_{i}$. Let us consider this in more detail. Every characteristic face $\Gamma$ of the polyhedron $\Delta=\Delta_{1}+\cdots+\Delta_{n}$ for a developed collection of polyhedra is locked, i.e. there are points among the components $\Gamma_{i}$ of its decomposition $\Gamma=\Gamma_{1}+\cdots+\Gamma_{n}$. Suppose that these are the components with indices $i_{1}, \ldots, i_{m}$, and let $a_{i_{1}}, \ldots, a_{i_{m}}$ be the coefficients of monomials corresponding to these points in the Laurent polynomials $P_{i_{1}}, \ldots, P_{i_{m}}$.

Lemma 2. If $m=1$ then either $R_{\Gamma}=a_{i_{1}}$ and $H_{\Gamma}$ is given by the equation $a_{i_{1}}=0$, or $H_{\Gamma}$ is empty. If $m>1$ then $H_{\Gamma}$ is empty a fortiori.

Proof. The condition of compatibility of the system $(\Gamma)$ in the torus $\left(\mathbb{C}^{*}\right)^{n}$ contains the relations $a_{i_{1}}=\cdots=a_{i_{m}}=0$, but, maybe, is not reducible to them. From this lemma 2 follows.

\section{$\S 7$. Reduction of the problem of finding the product of roots to the simplest case}

In this paragraph we will show how we can simplify the problem of finding the product of roots of a system of equations with fixed Newton polyhedra. In particular, we will reduce the problem of finding the product of roots of a system of equations with developed Newton polyhedra to the simplest case of binomial equations. For this purpose we introduce a new unknown - parameter $t$ and make the system homotopic in such a way that when $t \rightarrow \infty$ it asymptotically breaks down into a union of binomial systems.

We will need several new notations. Let $\tilde{\Delta}$ be an integral polyhedron in $\mathbb{R}^{n} \times \mathbb{R}^{1}$, let $P=\sum a_{k} x^{k}$ be a Laurent polynomial in $n$ variables, and let $G$ be any integral polyhedron in $\mathbb{R}^{n}$. Let us define the Laurent polynomial $P(\tilde{G})=\sum a_{k, l}(\tilde{G}) x^{k} t^{l}$ in the following way: the coefficient $a_{k, l}$ will be equal to zero, if either the point $(k, l)$ is not an upper point of the polyhedron $\tilde{\Delta}$, or the projection $k \in \mathbb{R}^{n}$ of this point does not belong to the polyhedron $G$. In the opposite case, if the point $(k, l)$ is an upper point of the polyhedron $\tilde{\Delta}$, and the point $k \in G$, we define $a_{k, l}(\tilde{G})=a_{k}$, where $a_{k}$ is the coefficient in the Laurent polynomial $P$ in front of the monomial $x^{k}$.

Suppose that

$$
P_{1}=\cdots=P_{n}=0
$$

is a system of equations in $\left(\mathbb{C}^{*}\right)^{n}$ and suppose that the supports of the Laurent polynomials $P_{1}, \ldots, P_{n}$ belong to the fixed Newton polyhedra $\Delta_{1}, \ldots, \Delta_{n}$. Assume that the system (1) is nondegenerate at infinity relatively to the polyhedra $\Delta_{1}, \ldots, \Delta_{n}$.

Suppose that $\Delta=\Delta_{1}+\cdots+\Delta_{n}$. Suppose further that $\tilde{\Delta}_{1}, \ldots, \tilde{\Delta}_{n}$, and $\tilde{\Delta}=$ $\tilde{\Delta}_{1}+\cdots+\tilde{\Delta}_{n}$ are integral polyhedra in $\mathbb{R}^{n} \times \mathbb{R}^{1}$, lying over the polyhedra $\Delta_{1}, \ldots, \Delta_{n}$. Suppose that $R\left(\Delta_{1}\right), \ldots, R\left(\Delta_{n}\right), R(\Delta)$ are the regular partitions of the polyhedra $\Delta_{1}, \ldots, \Delta_{n}, \Delta$ connected with the polyhedra $\tilde{\Delta}_{1}, \ldots, \tilde{\Delta}_{n}, \tilde{\Delta}$, and suppose that $S$ is the corresponding regular consistency of these partitions (see $\S 2$ ).

Theorem 1. Suppose that the following conditions are satisfied: 
1) for every polyhedron $\Gamma \subset R(\Delta)$ such that $\operatorname{dim} \Gamma<n$ the system $P_{1}\left(\Gamma_{1}\right)=$ $\cdots=P_{n}\left(\Gamma_{n}\right)=0$ is inconsistent in the torus $\left(\mathbb{C}^{*}\right)^{n}$, where $\Gamma_{i}$ is the $i$-th component of the $S$-decomposition of the polyhedron $\Gamma$;

2) for every characteristic face $G$ of the polyhedron $\Delta$ the system $P_{1}\left(\tilde{G}_{1}\right)=\cdots=$ $P_{n}\left(\tilde{G}_{n}\right)=0$ is inconsistent in the torus $\left(\mathbb{C}^{*}\right)^{n} \times\left(\mathbb{C}^{*}\right)$, where $G_{i} \subset \Delta_{i}$ is the $i$-th component of the decomposition of the face $G$.

Then the following equality holds.

$$
M\left(P_{1}, \ldots, P_{n}\right)=\prod_{s \in S} M\left(P_{1}\left(\Gamma_{1}(s)\right), \ldots, P_{n}\left(\Gamma_{n}(s)\right) .\right.
$$

Proof. 1. Without loss of generality we can assume that all the systems of equations which we consider in the proof are nondegenerate. Indeed, if the equality

$$
M\left(P_{1}, \ldots, P_{n}\right)=\prod_{s \in S} M\left(P_{1}\left(\Gamma_{1}(s)\right), \ldots, P_{n}\left(\Gamma_{n}(s)\right)\right.
$$

is proven for an open dense subset in the space of the systems satisfying the conditions of the theorem, then by continuity it also holds for all the systems satisfying the conditions of the theorem.

2. Let us consider the curve $X_{1}$ defined in $\left(\mathbb{C}^{*}\right)^{n} \times \mathbb{C}^{*}$ by the system of equations

$$
P_{1}\left(\tilde{\Delta}_{1}\right)=\cdots=P_{n}\left(\tilde{\Delta}_{n}\right)=0 .
$$

3. The curve $X_{1}$ does not have horizontal asymptots in $\left(\mathbb{C}^{*}\right)^{n} \times \mathbb{C}^{*}$. This follows from the condition 2) of inconsistency of all the systems

$$
P_{1}\left(\tilde{G}_{1}\right)=\cdots=P_{n}\left(\tilde{G}_{n}\right)=0
$$

for all the faces $G$ of the polyhedron $\Delta$ (see, for example, [6] about how to find the asymptotic power lines of a curve).

4. Let us also consider the curve $X_{2}$ which is the union over all the points $s \in S$ of the quasihomogeneous curves $X(s)$ defined by the equations $P_{1}(\tilde{\Gamma}(s))=\cdots=$ $P_{n}\left(\tilde{\Gamma}_{n}(s)\right)=0$.

5. The curves $X_{1}$ and $X_{2}=\bigcup_{s \in S} X(s)$ have exactly the same collection of asymptotic lines on which the coordinate $t$ approaches infinity. Indeed, this follows from the theory of Newton polyhedra (see, for example, [6]) according to which the asymptotic power lines of a curve are defined by the shortened systems

$$
P_{1}\left(\tilde{\Gamma}_{1}(s)\right)=\cdots=P_{n}\left(\tilde{\Gamma}_{n}(s)\right)=0 .
$$

The curve $X_{2}$ by definition is the union of such asymptotic power lines of the curve $X_{1}$ on which the coordinate $t$ approaches infinity.

6. According to theorem 3 from $\S 5$ for every $t_{0}$ the products in the group $\left(\mathbb{C}^{*}\right)^{n} \times \mathbb{C}^{*}$ of the points of intersection of the curve $X_{1}$ with the hyperplane $t=t_{0}$ and the points of intersection of the curve $X_{2}$ with the same hyperplane $t=t_{0}$ are equal. Letting in this equality $t=1$ and choosing projections on $\left(\mathbb{C}^{*}\right)^{n}$ of two equal products lying in $\left(\mathbb{C}^{*}\right)^{n} \times \mathbb{C}^{*}$, we obtain the equality

$$
M\left(P_{1}, \ldots, P_{n}\right)=\prod_{s \in S} M\left(P_{1}\left(\Gamma_{1}(s)\right), \ldots, P_{n}\left(\Gamma_{n}(s)\right) .\right.
$$


The theorem is proven.

If the condition 1) of theorem 1 holds for almost all the systems of equations (1), then with the condition 2) of theorem 2 the business is worse. Of course, for every characteristic face $G$ of the polyhedron $\Delta$ the system

$$
P_{1}\left(G_{1}\right)=\cdots=P_{n}\left(G_{n}\right)=0
$$

in $\left(\mathbb{C}^{*}\right)^{n}$ is almost always inconsistent. The point is that the polyhedra $G_{1}, \ldots, G_{n}$ can be parallelly translated in a $(n-1)$-dimensional subspace of $\mathbb{R}^{n}$. In the result we obtain an equivalent system with $n$ equations which basically depends on a number of unknowns which is smaller than $n$. However, if we add one more unknown $t$, then the resulting system

$$
P_{1}\left(\tilde{G}_{1}\right)=\cdots=P_{n}\left(\tilde{G}_{n}\right)=0
$$

can turn out, and, in the general case, does in fact turn out, consistent. The condition 2, generally speaking, imposes considerable restrictions on the choice of how to introduce the parameter $t$. These conditions will be observed if, for example, not to introduce at all the parameter $t$ in the monomials of the Laurent polynomials $P_{i}$ which correspond to the boundary points of the polyhedra $\Delta_{i}$. In other words, if to choose the polyhedra $\tilde{\Delta}_{1}, \ldots, \tilde{\Delta}$ in $\mathbb{R}^{n} \times \mathbb{R}^{1}$ in such a way that the vertices of the polyhedra $\tilde{\Delta}_{i}$, lying above the vertices of the polyhedra $\Delta_{i}$, belonged to the hyperplane $\mathbb{R}^{n} \times(0)$. For developed collections of polyhedra $\Delta_{1}, \ldots, \Delta_{n}$ the condition 2) in theorem 1 is satisfied automatically. In the formulation of the next corollary we suppose that the polyhedra $\Delta_{1}, \ldots, \Delta_{n}$ are the Newton polyhedra of the Laurent polynomials $P_{1}, \ldots, P_{n}$, i.e. that the coefficients of the Laurent polynomials $P_{i}$ in front of monomials corresponding to the vertices of polyhedra $\Delta_{i}$ are nonzero.

Corollary 1. Let

$$
P_{1}=\cdots=P_{n}=0
$$

be a system of equations in $\left(\mathbb{C}^{*}\right)^{n}$ nondegenerate at infinity, for which the collection of Newton polyhedra $\Delta_{1}, \ldots, \Delta_{n}$ is developed. Suppose that $\tilde{\Delta}_{1}, \ldots, \tilde{\Delta}_{n}$ are integral polyhedra lying over the polyhedra $\Delta_{1}, \ldots, \Delta_{n}$, and suppose that $\mathcal{R}\left(\Delta_{1}\right), \ldots, \mathcal{R}\left(\Delta_{n}\right), \mathcal{R}(\Delta)$ are the corresponding regular partitions with the regular consistency $S$. Suppose that for every polyhedron $\Gamma \subset \mathcal{R}(\Delta)$ such that $\operatorname{dim} \Gamma<n$ the system of equations $P_{1}\left(\Gamma_{1}\right)=\cdots=P_{n}\left(\Gamma_{n}\right)=0$ is inconsistent in the torus $\left(\mathbb{C}^{*}\right)^{n}$, where $\Gamma_{i}$ is the $i$-th component of the $S$-decomposition of the polyhedron $\Gamma$. Then we have the following equality:

$$
M\left(P_{1}, \ldots, P_{n}\right)=\prod_{s \in S} M\left(P_{1}(s), \ldots, P_{N}(s)\right) .
$$

Proof. In the hypothesis of corollary 1, the condition 2) of theorem 1 is satisfied automatically. Indeed, by hypothesis, every characteristic face $G$ of the polyhedron $\Delta$ is locked. This means that among the components of its decomposition $G=$ $G_{1}+\cdots+G_{n}$ there is a component $G_{j}=k$ which is a point - a vertex of the polyhedron $\Delta_{j}$. By hypothesis, the coefficient $a_{j k}$ in front of the monomial $x^{k}$ in 
the Laurent polynomial $P_{j}$ is not equal to zero. Therefore the equation $P_{j}\left(\tilde{G}_{j}\right)=0$ will have the from $a_{i j} x^{k} t^{l}=0$ and it will be inconsistent in the torus $\left(\mathbb{C}^{*}\right)^{n} \times \mathbb{C}^{*}$. So corollary 1 follows from theorem 1 .

Sometimes it is useful to apply corollary 1 not to the system of equations itself, but to a somewhat modified system. Suppose that $\tilde{\tilde{\Delta}}_{1}, \ldots, \tilde{\Delta}_{n}$ is a collection of polyhedra lying over the polyhedra $\Delta_{1} \ldots, \Delta_{n}$ such that any consistent collection $\Gamma_{1}(s), \ldots, \Gamma_{n}(s)$ of polyhedra from the corresponding regular partitions $\mathcal{R}\left(\Delta_{1}\right), \ldots, \mathcal{R}\left(\Delta_{n}\right)$ is affinely independent. Let us associate with the partitions $\mathcal{R}\left(\Delta_{1}\right), \ldots, \mathcal{R}\left(\Delta_{n}\right)$ the collection of Laurent polynomials $T_{1}, \ldots, T_{n}$ whose coefficients are defined by the following rule. The coefficient $T_{i k}$ of the Laurent polynomial $T_{i}$ in front of the monomial $x^{k}$ is equal to: zero, if the point $k$ is not a vertex of the partition $\mathcal{R}\left(\Delta_{i}\right)$; the coefficient $P_{i k}$ in front of the monomial $x^{k}$ in the Laurent polynomial $P_{i}$, if the point is a vertex of the polyhedron $\Delta_{i}$; one, in every other case, i.e. if the point $k$ is not a vertex of the polyhedron $\Delta_{i}$, but is one of the vertices of the partition $\mathcal{R}\left(\Delta_{i}\right)$ of this polyhedron.

Theorem 2. For a system of equations $P_{1}=\cdots=P_{n}=0$ in $\left(\mathbb{C}^{*}\right)^{n}$ with developed collection of Newton polyhedra $\Delta_{1}, \ldots, \Delta_{n}$, and for a collection of polyhedra $\tilde{\Delta}_{1}, \ldots, \tilde{\Delta}_{n}$ defining a regular partition of the polyhedra $\Delta_{1}, \ldots, \Delta_{n}$ in such a way that any collection of consistent polyhedra $\Gamma_{1}(s), \ldots, \Gamma_{n}(s)$ is affinely-independent, the following formula holds:

$$
M\left(P_{1}, \ldots, P_{n}\right)=\prod_{s \in S} M\left(T_{1}\left(\Gamma_{1}(s)\right), \ldots, T_{n}\left(\Gamma_{n}(s)\right)\right) .
$$

Proof. 1. The products of the roots in $\left(\mathbb{C}^{*}\right)^{n}$ of the systems of equations $P_{1}=\cdots=$ $P_{n}=0$ and $T_{1}=\cdots=T_{n}=0$ are the same. Indeed, these systems have exactly the sane developed collection of Newton polyhedra and the same coefficients at the vertices.

2. For every polyhedron $\Gamma \subset \mathcal{R}(\Delta)$ of dimension smaller than $n$ among the components $\Gamma_{1}(s), \ldots, \Gamma_{n}(s)$ of its $S$-decomposition there is necessarily a vertex. This follows from the affine independence of the polyhedra $\Gamma_{1}(s), \ldots, \Gamma_{n}(s)$. Suppose that $\Gamma_{i}(s)$, one of such components, is an itegral point $k \in \Delta_{i}$. The coefficient $T_{i k}$ in front of the monomial $x^{k}$ is nonzero by construction (it is equal to one, if $k$ is not a vertex of the polyhedron $\Delta_{i}$, or it is equal to the nonzero coefficient $a_{i k}$ of the Laurent polynomial $P_{i}$, if $k$ is a vertex of the polyhedron $\Delta_{i}$ ). Therefore the system $T_{1}\left(\Gamma_{1}(s)\right)=\cdots=T_{n}\left(\Gamma_{n}(s)\right)=0$ in $\left(\mathbb{C}^{*}\right)^{n}$ is inconsistent. Using corollary 1 concludes the proof.

Theorem 2 reduces the problem of calculating $M\left(P_{1}, \ldots, P_{n}\right)$ for systems of equations with a developed collection of Newton polyhedra to the following two problems.

Problem 1. The problem of calculating the product $M\left(T_{1}(s), \ldots, T_{n}(s)\right)$ for binomial systems of equations.

In the situation of theorem 2 every consistent system $T_{1}\left(\Gamma_{1}(s)\right)=\cdots=T_{n}\left(\Gamma_{n}(s)\right)=$ 0 can be only binomial. From the condition of the affine independence it follows that either there are points among the polyhedra $\Gamma_{i}(s)$ and then the corresponding of equations is inconsistent, or all the polyhedra $\Gamma_{1}(s), \ldots, \Gamma_{n}(s)$ are segments. 
By construction the Laurent polynomials $T_{i}\left(\Gamma_{i}(s)\right)$ in this case contain only two monomials with nonzero coefficients which correspond to the vertices of the segment $\Gamma_{i}(s)$. The problem of calculating the product of roots of a binomial system of equations is not difficult. In fact, such a system can be even solved explicitly. It is especially easy to find the components of the product up to a sign. However, we need the complete answer, which must be also in a convenient form. Therefore we will consider the problem 1 in detail in the following paragraphs.

Problem 2. The problem of calculating the products of the found elements $M\left(T_{1}\left(\Gamma_{1}(s)\right), \ldots, T_{n}\left(\Gamma_{n}(s)\right)\right)$ over all the consistent systems of binomial equations.

If the answer to the problem 1 is written in a convenient form, then, as we will see later, the problem 2 can be easily solved with the help of the technique of combinatorial coefficients (developed in $\S \S 1-4$.)

\section{$\S 8$. The product of preimages of a homomorphism}

Let $\tau$ be a homomorphism of a commutative group $G_{1}$ onto a commutative group $G_{2}$ and suppose that the kernel of $\tau$ contains a finite number $N$ of elements. Using the homomorphism $\tau$ let us construct the map $M: G_{2} \rightarrow G_{1}$ which associates to every point $a$ in the group $G_{2}$ the element $M(a)$ in the group $G_{1}$ which is equal to the product of all the preimages of the point $a$ under the map $\tau$. How does the element $M(a)$ depend on the point $a$ and the homomorphism $\tau$ ? In this paragraph we discuss this question and give a full answer in the case of homomorphisms of groups $G_{1}$ and $G_{2}$ isomorphic to a $n$-dimensional torus $\left(\mathbb{C}^{*}\right)^{n}$.

Lemma 1. For every point $a \in G_{2}$, the $N$-th power of its preimage does not depend on the choice of the preimage. The map $a \mapsto\left(\tau^{-1}(a)\right)^{N}$ defines a homomorphism from the group $G_{2}$ to the group $G_{1}$.

Proof. Two different preimages $b_{1}$ and $b_{2}$ of the point $a$ differ by a preimage $q$ of the element $e, b_{1}=b_{2} q, \tau(q)=e$. For every element $q$ we have $q^{N}=e$ since by hypothesis the group $\operatorname{ker} \tau$ contains $N$ elements. Moreover, if an element $b_{1}$ is one of the preimages of a point $a_{1}$, and an element $b_{2}$ is one of the preimages of a point $a_{2}$, then the element $b_{1} b_{2}$ is one of the preimages of the product $a_{1} a_{2}$. Therefore the map $a \mapsto\left(\tau^{-1}(a)\right)^{N}$ is a homomorphism.

The following lemma is just as elementary.

Lemma 2. We have the equality $M=\left(\tau^{-1}\right)^{N} \cdot C$, where $C$ is some element of the group $G_{1}$. Therefore for every pair of points $a_{0}, x \in G_{2}$ we have the equality $M\left(a_{0} x\right)=M\left(a_{0}\right)\left(\tau^{-1}(x)\right)^{N}$.

Let us now compute the map $\left(\tau^{-1}\right)^{N}$ for a homomorphism $\tau$ from the torus $G_{1}=\left(\mathbb{C}^{*}\right)^{n}$ to another torus $G_{2}=\left(\mathbb{C}^{*}\right)^{n}$. Such homomorphism is given by an integer-values real linear map $A_{\tau}$ from the Lie algebra $\mathfrak{g}_{1}$ of the torus $G_{1}$ to the Lie algebra $\mathfrak{g}_{2}$ of the torus $G_{2}$. The condition of being integral-valued consists in the fact that the operator $A_{\tau}$ maps the lattice of algebraic one-parameter groups $\mathbb{Z}^{n} \subset \mathfrak{g}_{1}$ of the first torus to the lattice of algebraic one-parameter groups of the second torus. On real subspaces of the algebras $\mathfrak{g}_{1}$ and $\mathfrak{g}_{2}$ a Haar's measure is defined; it is normalized by the following condition: the value of the measure on a parallelepiped generated by any basis in the lattice $\mathbb{Z}^{n}$ of one-parameter groups is equal to one.

It is easy to check the following 
Lemma 3. The homomorphism $\tau:\left(\mathbb{C}^{*}\right)^{n} \rightarrow\left(\mathbb{C}^{*}\right)^{n}$ has a finite number of preimages if and only if the linear map $A_{\tau}: \mathfrak{g}_{1} \rightarrow \mathfrak{g}_{2}$ is nondegenerate. The number $N$ of preimages of the homomorphism $\tau$ is equal to $\left|\operatorname{det} A_{\tau}\right|=A_{\tau}^{*} \mu_{2} / \mu_{1}$. The homomorphism $\left(\tau^{-1}\right)^{N}: G_{2} \rightarrow G_{1}$ is given by the linear map

$$
\left|\operatorname{det} A_{\tau}\right| A_{\tau}^{-1}: \mathfrak{g}_{2} \rightarrow \mathfrak{g}_{1} .
$$

Let us now write the map $\left(\tau^{-1}\right)^{N}$ in coordinates. Let us fix the representation of the tori $G_{1}$ and $G_{2}$ in the form of the Descartes products of the groups $\mathbb{C}^{*}$ with some fixed characters (coordinates) on them.

After this fixation every point $x$ of the torus $G_{1}$ is defined by a collection of nonzero numbers $x=x_{1}, \ldots, x_{n}$; every point $c$ of the torus $G_{2}$ is defined by a collection of nonzero numbers $c=c_{1}, \ldots, c_{n}$; the homomorphism $\tau$ is defined by a collection of characters $\chi_{1}, \ldots, \chi_{n}$, where $\tau(x)=\chi_{1}(x), \ldots, \chi_{n}(x)$.

To the characters $\chi_{1}, \ldots, \chi_{n}$ correspond integral points $k_{1}, \ldots, k_{n}$ in the lattice of characters $\mathbb{Z}^{n}$ such that $\chi_{i}(x)=x^{k_{i}}=x_{1}^{k_{i 1}} \cdots x_{n}^{k_{i n}}$, where $k_{i}=k_{i 1}, \ldots, k_{i n}$, $i=1, \ldots, n$. The kernel of the homomorphism $\tau$ contains $|\operatorname{det} K|$ elements, where $K$ is the matrix with the elements $k_{i j}$. We want to calculate the element $\left(\tau^{-1}(c)\right)^{N}$ in the group $G_{1}$. Let us fix the character $\chi=x^{k_{n+1}}, k_{n+1}=k_{n+1,1}, \ldots, k_{n+1, n}$, we will calculate the number $\chi\left(\left(\tau^{-1}(c)\right)^{N}\right.$. It is clear that the calculation of such number for every character $\chi$ is equivalent to the calculation of the point $\left(\tau^{-1}(c)\right)^{N}$. The following lemma 4 is a reformulation of lemma 3 . In this reformulation we use the coordinates and instead of the map $A_{\tau}$ we use the adjoint map $K=A_{\tau}^{*}$.

Lemma 4. The number $\chi\left(\tau^{-1}(c)\right)^{|\operatorname{det} K|}$ is equal to

$$
c_{1}^{\operatorname{det}\left(k_{n+1}, k_{2}, \ldots, k_{n}\right) \operatorname{sign} \operatorname{det}(K)} \ldots c_{n}^{\operatorname{det}\left(k_{1}, \ldots, k_{n-1}, k_{n+1}\right) \operatorname{sign} \operatorname{det}(K)},
$$

where $\tau=x^{k_{1}}, \ldots, x^{k_{n}}, \chi=x^{k_{n+1}}, c=c_{1}, \ldots, c_{n}$.

Let us return to the general situation. To apply lemma 2 we need to calculate $M\left(a_{0}\right)$ for some fixed point $a_{0}$. For points $a_{0} \in G_{2}$ for which $a_{0}^{2}=e$ the problem is significantly simpler.

In the groups $G_{1}$ and $G_{2}$ let us consider the subgroups $R_{1}$ and $R_{2}$ consisting of the elements whose square is equal to $e$. The intersection of the group $R_{1}$ with the kernel of the homomorphism $\tau$ contains a finite number of elements.

Let $\tau_{0}: R_{1} \rightarrow R_{2}$ be the restriction of the homomorphism $\tau$ on the group $R_{1}$. The homomorphism $\tau_{0}$, generally speaking, is not "onto." Let us denote by Trace $\tau_{\tau_{0}}(a)$ the product of all the preimages of a point $a \in R_{2}$ under the homomorphism $\tau_{0}$. If the point $a$ does not have any preimages, then let Trace $\tau_{\tau_{0}}(a)=e$.

Lemma 5. For any point $a \in R_{2}$ the following equality holds:

$$
\text { Trace }_{\tau_{0}}(a)=M(a) \text {. }
$$

Proof. The set $\tau^{-1}(a)$ is invariant with respect to the action of involution $G_{1} \rightarrow$ $G_{1}^{-1}$. Indeed, if $\tau(b)$ belongs to the group $R_{2}$, then $\tau\left(b^{-1}\right)=\tau(b)$ since every element in the group $R_{2}$ is equal to its inverse element. The set $\tau_{0}^{-1}(a)$ if fixed by the action of involution $G_{1} \rightarrow G_{1}^{-1}$ since every element in the group $R_{1}$ is equal to its inverse element. Furthermore, if the elements $b$ and $b^{-1}$ in the set $\tau^{-1}(a)$ do not coincide, then under multiplication they cancel each other. 
Lemma 6. Let $R$ be a finite abelian group such that all of its elements have order two. Then if the number of elements in the group $R$ is not equal to two, then the product if all the elements in the group $R$ is equal to $e$. If this group contains exactly two elements, then the product is equal to the non-identity element of this group.

Proof. The group $R$ is isomorphic to a group $\mathbb{Z}_{2}^{m}$. Therefore if the group $R$ contains more than two elements, then it can be represented in the form of the product $R=P \times Q$ of two non-identity groups $P$ and $Q$. It is clear that

$$
\prod_{a \in R} a=\left(\prod_{b \in P} b\right)^{q} \times\left(\prod_{c \in Q} c\right)^{p},
$$

where $p$ and $q$ are correspondingly the numbers of elements in the groups $P$ and $Q$. In this case the numbers $p$ and $q$ are even, while all the elements $b$ and $c$ have order 2. Lemma 6 is proven.

Suppose that the groups $R_{1}$ and $R_{2}$ are isomorphic to the group $\mathbb{Z}_{2}^{m}$, and suppose that $\tau: R_{1} \rightarrow R_{2}$ is a homomorphism of these groups. Using the homomorphism $\tau$ let us construct the map Trace ${ }_{\tau}: R_{2} \rightarrow R_{1}$ assigning to each point $a$ the product of its preimages. We do not assume here that the map $\tau$ is "onto." The product of an empty set of elements in the definition of the map Trace ${ }_{\tau}$ we will consider equal to $e$.

Lemma 7. For a homomorphism $\tau: R_{1} \rightarrow R_{2}$, the map Trace $\tau: R_{2} \rightarrow R_{1}$ is computed in the following way:

1) if $\operatorname{ker} \tau=e$, then $\operatorname{Trace}_{\tau}=\tau^{-1}$;

2) if the group $\operatorname{ker} \tau$ contains more than two elements, then Trace $_{\tau}=e$;

3) if the group $\operatorname{ker} \tau$ contains exactly two elements, then on the image of the group $R_{1}$ the map Trace ${ }_{\tau}$ is equal to the non-identity element of the group $\operatorname{ker} \tau$, and on the complement to the image of the group $R_{1}$ the map Trace $\tau$ is equal to the identity element $e$.

The proof is obtained by applying lemma 6 to the group $R=\operatorname{ker} \tau$.

In the calculations in $\S 11$ we will need the case when the groups $R_{1}$ and $R_{2}$ are the subgroups of standard $n$-dimensional tori $G_{1}$ and $G_{2}$ which contain all the elements of order two in these tori. We will identify the elements $x \in R_{1}$ and $c \in R_{2}$ of these groups with the points $x=x_{1}, \ldots, x_{n}, c=c_{1}, \ldots, c_{n}$, all coordinates of which are equal to \pm 1 . To every homomorphism $\tau: G_{1} \rightarrow G_{2}$ of the standard tori $G_{1}$ and $G_{2}$ corresponds its restriction on the group $R_{1}$ which we will also denote by the same symbol $\tau, \tau: R_{1} \rightarrow R_{2}$. In the calculations in $\S 11$ we will need the following

Definition 1. Let us denote by $\mathbf{F}\left(k_{1}, \ldots, k_{n}\right)$ the function which assigns to a collection of $n$ integral points $k_{1}, \ldots, k_{n}$ in the lattice $\mathbb{Z}^{n}$ the element of the group $R_{1} \subset\left(\mathbb{C}^{*}\right)^{n}$ defined by the formula

$$
\mathbf{F}\left(k_{1}, \ldots, k_{n}\right)=\operatorname{Trace}_{\tau}(-\mathbf{1}) .
$$

In this formula $\tau$ is the restriction on the group $R_{1}$ of the homomorphism $\tau:\left(\mathbb{C}^{*}\right)^{n} \rightarrow$ $\left(\mathbb{C}^{*}\right)^{n}$ given by the formulas $c_{1}=x^{k_{1}}, \ldots, c_{n}=x^{k_{n}}$, and $-\mathbf{1}$ is the element of the group $R_{2} \subset\left(\mathbb{C}^{*}\right)^{n}$ all of whose components are equal to -1 , i.e. $-\mathbf{1}=(-1, \ldots,-1)$. 
Lemma 7 contains the complete description of the function $\mathbf{F}\left(k_{1}, \ldots, k_{n}\right)$. The coordinates of a point $\mathbf{F}\left(k_{1}, \ldots, k_{n}\right)$ will be written explicitly later in corollary 1.

Let us now turn to writing the map Trace $\tau$ in coordinates. The restriction of the character $x^{k}$ of the group $\left(\mathbb{C}^{*}\right)^{n}$ on the subgroup $R_{1}$ depends not on the vector $k \in \mathbb{Z}^{n}$ itself, but on its image in the quotient group $\mathbb{Z}^{n} / 2 \mathbb{Z}^{n}$. Let $\tau: R_{1} \rightarrow R_{2}$ be the restriction of the homomorphism $\tau: \rightarrow\left(\mathbb{C}^{*}\right)^{n}$ given the formulas $c_{1}=$ $x^{k_{1}}, \ldots, c_{n}=x^{k_{n}}$. To describe the point Trace $\tau$ let us consider one more character $x^{k_{n+1}}$ and let us compute its value on this point. We will consider the vectors $k_{1}, \ldots, k_{n}, k_{n+1}$ as elements of the vector space $\mathbb{Z}^{n} / 2 \mathbb{Z}^{n}$ over the field $\mathbb{Z} / 2 \mathbb{Z}$.

The following lemma 8 is the coordinate description of lemma 7 .

Lemma 8. For a homomorphism $\tau: R_{1} \rightarrow R_{2}$ given by the collection of characters $k_{1}, \ldots, k_{n}$, for any character $k_{n+1}$ on the group $R_{1}$, and for any point $c \in R_{2}$ the value of the character $k_{n+1}$ on the point $\operatorname{Trace}_{\tau}(c)$ is computed as follows:

1) if the rank of the vectors $k_{1}, \ldots, k_{n+1}$ in the vector space $\mathbb{Z}^{n} / 2 \mathbb{Z}^{n}$ over the field $\mathbb{Z} / 2 \mathbb{Z}$ is smaller than $n$, then $k_{n+1}\left(\operatorname{Trace}_{\tau}(c)\right)=1$;

2 ) if the vectors $k_{1}, \ldots, k_{n+1}$ in $\mathbb{Z}^{n} / 2 \mathbb{Z}^{n}$ are related by the unique relation $\lambda_{1} k_{1}+$ $\cdots+\lambda_{n+1} k_{n+1}=0, \lambda_{i} \in \mathbb{Z} / 2 \mathbb{Z}$, then

$$
k_{n+1}\left(\operatorname{Trace}_{\tau}(c)\right)=(-1) c_{1}^{\lambda_{1}} \ldots c_{n}^{\lambda_{n}}(-1)^{\lambda_{n+1}},
$$

where $c_{1}, \ldots, c_{n}$ are the coordinates of the point $c$.

It is sufficient to calculate $\operatorname{Trace}_{\tau}(c)$ for only one point $c$ (see lemma 2). From lemma 8 it is clear that the preimage of the point $c=\mathbf{- 1}$ depends on the characters $k_{1}, \ldots, k_{n+1}$ in the most symmetrical way. We will write the answer separately for this point.

For the formulation of the answer we will need the definition of a remarkable function $F$ of $(n+1)$ integral vectors $k_{1}, \ldots, k_{n}, k_{n+1} \in \mathbb{Z}^{n}$ with values in $\mathbb{Z} / 2 \mathbb{Z}$. This function $F$ does not depend on the vectors $k_{1}, \ldots, k_{n}, k_{n+1}$ themselves, but rather on their classes $\bar{k}_{1}, \ldots, \bar{k}_{n}, \bar{k}_{n+1}$ modulo 2 . In the next paragraph we will describe more explicitly the role of the function $F$ in linear algebra over the field $\mathbb{Z} / 2 \mathbb{Z}$.

\section{Definition 2.}

$F\left(k_{1}, \ldots, k_{n}, k_{n+1}\right)=\left\{\begin{array}{l}0, \quad \text { if the rank of the system of vectors } \bar{k}_{1}, \ldots, \bar{k}_{n}, \bar{k}_{n+1} \\ \text { over the field } \mathbb{Z} / 2 \mathbb{Z} \text { is not equal to } n, \\ \left(\lambda_{1}+\cdots+\lambda_{n+1}+1\right) \text { mod } 2, \quad \text { if the vectors } \\ \bar{k}_{1}, \ldots, \bar{k}_{n}, \bar{k}_{n+1} \text { are related over the field } \mathbb{Z} / 2 \mathbb{Z} \\ \text { by a unique relation } \\ \lambda_{1} \bar{k}_{1}+\cdots+\lambda_{n+1} \bar{k}_{n+1} \equiv 0 \bmod 2 .\end{array}\right.$

Corollary 1. Suppose that a homomorphism $\tau$ is given by the collection of characters $k_{1}, \ldots, k_{n}$. Then $k_{n+1}\left(\mathbf{F}\left(k_{1}, \ldots, k_{n}\right)\right)=(-1)^{F\left(k_{1}, \ldots, k_{n}, k_{n+1}\right)}$.

Let us consider the system of binomial equations in $\left(\mathbb{C}^{*}\right)^{n}$

$$
\begin{gathered}
Q_{1} x^{q_{1}}+R_{1} x^{r_{1}}=0, \\
\vdots \\
Q_{n} x^{q_{n}}+R_{n} x^{r_{n}}=0,
\end{gathered}
$$


where $Q_{i}, R_{i}$ are nonzero complex numbers, $q_{i}, r_{i}$ are vectors in the lattice $\mathbb{Z}^{n}$, $x=x_{1}, \ldots, x_{n}, x^{q_{i}}$ and $x^{r_{i}}$ are monomials. Denote by $\operatorname{det} K$ the determinant of the matrix composed of the vectors $k_{i}=q_{i}-r_{i}$. Suppose that $k \in \mathbb{Z}^{n}$ is an integral vector and $\chi_{k}=x^{k}$ is the character of the torus $\left(C^{*}\right)^{n}$ which corresponds to this vector.

Theorem 1. Suppose that the vectors $k_{i}$ are linearly independent. Then the value of the character $\chi_{k}$ on the product in the group $\left(\mathbb{C}^{*}\right)^{n}$ of all the solutions $a_{j}$ of the system (1) is calculated by the following formula:

$$
\chi_{k}\left(\prod a_{j}\right)=(-1)^{F\left(k_{1}, \ldots, k_{n}, k\right)}\left(\frac{R_{1}}{Q_{1}}\right)^{\operatorname{det}\left(k, k_{2}, \ldots, k_{n}\right) \operatorname{sign} \operatorname{det} K} \ldots .
$$

Proof. The original system of the binomial equations can be rewritten in the form

$$
\begin{gathered}
x^{k_{1}}=-\frac{R_{1}}{Q_{1}}, \\
\vdots \\
x^{k_{n}}=-\frac{R_{n}}{Q_{n}} .
\end{gathered}
$$

Thus, in the theorem we are talking about calculating the value of the character $k_{n+1}=k$ on the product of all the preimages of the point $(-\mathbf{1}) c$, where $c=\frac{R_{1}}{Q_{1}}, \ldots, \frac{R_{n}}{Q_{n}}$, under the homomorphism $\tau$ given by the collection of characters $k_{1}, \ldots, k_{n}$. All we need to do is to use lemma 2 for $a_{0}=-\mathbf{1}$, lemma 4 , and corollary 1 .

Remark. For a particular symmetric system (1), for which all the coefficients $Q_{i}$ and $R_{i}$ are equal to 1 , it is required to compute the product of the preimages of the point $c=-\mathbf{1}$. This explains why the value of the product $M(a)$ is particularly simple for the point $a=-\mathbf{1}$.

\section{§. Analog of the determinant for $(n+1)$ vectors in a $n$-dimensional space over the field $\mathbb{Z} / 2 \mathbb{Z}$}

A determinant of $n$ vectors in a $n$-dimensional linear space over the field $\mathbb{Z} / 2 \mathbb{Z}$ is the unique nonzero multilinear function of $n$ vectors (taking values in the field $\mathbb{Z} / 2 \mathbb{Z}$ ) which is invariant relative to linear transformations and equal to zero if the rank of $n$ vectors is smaller than $n$.

It turns out that there exists a unique function of $(n+1)$ vectors in a $n$ dimensional space over the field $\mathbb{Z} / 2 \mathbb{Z}$ which possesses exactly the same properties. In this paragraph we discuss this function in detail. We encountered it in theorem 1 of the previous paragraph, it appears in Parshin-Kato* theory ([2], [10]), it will be required to us in the formulation of the theorem in $\S 11$. Let us repeat the definition of this function.

*As I found from A. N. Parshin, the function $\bar{F}$ and the related linear algebra weren't know to him, however exactly the same function occurs in the symbols he created. 
Definition. Let us denote by $\bar{F}$ the following function of $(n+1)$ vectors in a $n$-dimensional linear space over the field $\mathbb{Z} / 2 \mathbb{Z}, \bar{F}$ takes values in $\mathbb{Z} / 2 \mathbb{Z}$ :

a) the function $\bar{F}\left(k_{1}, \ldots, k_{n+1}\right)$ is equal to zero, if the rank of the collection of vectors $k_{1}, \ldots, k_{n+1}$ is smaller than $n$;

b) the function $\bar{F}\left(k_{1}, \ldots, k_{n+1}\right)$ is equal to $\lambda^{1}+\cdots+\lambda^{n+1}+1$, if the vectors $k_{1}, \ldots, k_{n+1}$ are related by the unique relation $\lambda^{1} k_{1}+\cdots+\lambda^{n+1} k_{n+1}=0$.

Lemma 1. The function $\bar{F}$

1) is $G L_{n}(\mathbb{Z} / 2 \mathbb{Z})$-invariant, i.e. for any linear transformation $A \in G L_{n}(\mathbb{Z} / 2 \mathbb{Z})$ the equality $\bar{F}\left(k_{1}, \ldots, k_{n+1}\right)=\bar{F}\left(A k_{1}, \ldots, A k_{n+1}\right)$ holds;

2 ) is equal to zero on the collections of vectors $k_{1}, \ldots, k_{n+1}$ whose rank is smaller than $n$;

3) is multilinear.

Proof. The properties 1 and 2 are basically contained in the definition of the function $\bar{F}$. For the proof of the property 3 , it is sufficient to show that for every fixed collection of vectors $k_{1}, \ldots, k_{n}$ the function $\varphi(k)=\bar{F}\left(k_{1}, \ldots, k_{n}, k\right)$ is linear.

The rank of the system of vectors $k_{1}, \ldots, k_{n}$ can be equal to $n$, be equal to $n-1$, or be smaller than $n-1$. Let us consider these three cases separately.

1. The rank is equal to $n$. In this case the vectors $k_{1}, \ldots, k_{n}$ form a basis in the linear space and the vector $k$ can be represented in a unique way as their linear combination $k=\lambda_{1} k_{1}+\cdots+\lambda_{n} k_{n}$. Then $\varphi(k)=\bar{F}\left(k_{1}, \ldots, k_{n}, k\right)=\left(\lambda_{1}+\cdots+\lambda_{n}\right)$ mod 2 and so is a linear function of $k$.

2. The rank is equal to $n-1$. In this case the function $\varphi$ vanishes on the hyperplane $\Lambda$ spanned by the vectors $k_{1}, \ldots, k_{n}$, and it assumes a constant value on the complement to this hyperplane. Indeed, if $k \in \Lambda$, then the rank of the system of vectors $k_{1}, \ldots, k_{n}, k$ is smaller than $n$, so the function $\bar{F}\left(k_{1}, \ldots, k_{n}, k\right)$ is equal to zero. If $k \notin \Lambda$, then the vectors are related by a unique relation not depending on the vector $k$, hence the function $\varphi$ is constant on the complement to the hyperplane $\Lambda$. The function which possesses such property in a linear space over the field $\mathbb{Z} / 2 \mathbb{Z}$ is clearly linear.

3 . The rank is smaller than $n-1$. In this case the function $\varphi(k)$ is identically equal to zero, and, therefore, it is a linear function of $k$.

Lemma 2. There exists a unique nonzero function $\bar{F}$ which satisfies the properties 1-3 of lemma 1.

Proof. It is enough to define a multilinear function on all collections $e_{i_{1}}, \ldots, e_{i_{n+1}}$ of vectors from the standard basis $e_{1}, \ldots, e_{n}$. From the property 2 it follows that the function can be not equal to zero only if in the collection all the vectors except for two are different. From the property 1 it follows that on all such collections the function $\bar{F}$ takes the same value. If this value is equal to zero, then the function $\bar{F}$ is zero. The only other remaining possibility is that this value is equal to one. This possibility corresponds to the function $\bar{F}$ defined above, which according to lemma 1 is indeed $G L_{n}(\mathbb{Z} / 2 \mathbb{Z})$-invariant.

Lemma 3. In coordinates the function $\bar{F}$ can be expressed by the formula

$$
\bar{F}\left(k_{1}, \ldots, k_{n+1}\right)=\sum_{j>i} \Delta_{i j}
$$


where $\Delta_{i j}$ is the determinant of the $(n \times n)$ matrix whose first $(n-1)$ columns represent the sequence of vectors $k_{1}, \ldots, k_{n+1}$ from which the vectors with indices $i$ and $j$ are deleted, and the last column is the coordinate-wise product of the vectors $k_{i}$ and $k_{j}$.

Proof. The function $\sum_{j>i} \Delta_{i j}$ is a multilinear function of vectors $k_{1}, \ldots, k_{n+1}$. On collections of vectors from the standard basis it clearly coincides with the function $\bar{F}$.

Let us mention another explicit formula for the function $\bar{F}$.

Let us begin with the explicit description of the relationship between $(n+1)$ vectors in a $n$-dimensional space. To define an ordered collection of $(n+1)$ vectors $k_{1}, \ldots, k_{n+1}$ in the $n$-dimensional linear space $L^{n}$ is the same thing as to define a linear map $A: L^{n+1} \rightarrow L^{n}$ for which $A\left(e_{i}\right)=k_{i}$, where $e_{1}, \ldots, e_{n+1}$ is the standard basis in $L^{n+1}$.

The dual map $A^{*}: L^{n *} \rightarrow L^{(n+1) *}$ is given by the ordered collection of $n$ covectors - the images of the basis covectors $v_{i}^{*}$. Using the collection of covectors $A^{*} v_{i}^{*}$ we can define a vector $\lambda \in L^{n+1}$ which is the vector product of these vectors. (To define the vector product we need a volume form. We assume that the volume form is fixed in such a way that its value $\omega\left(e_{1}^{*}, \ldots, e_{n+1}^{*}\right)$ on the dual basis is equal to 1 . The vector $\lambda$ is now defined by the relation $\left\langle\lambda, a^{*}\right\rangle=\omega\left(a^{*} \wedge A v_{1}^{*} \wedge \cdots \wedge A v_{n}^{*}\right)$, which should be true for any covector $a^{*}$.)

In the basis $e_{1}, \ldots, e_{n+1}$ the $i$-th component of the vector $\lambda$ is equal to the determinant which is obtained by removing the $i$-th row from the matrix composed of the vectors $k_{1}, \ldots, k_{n+1}$. We have the following obvious relation:

$$
\sum \Delta_{i} k_{i}=0
$$

Lemma 4. The function $\prod_{1 \leq i \leq n+1}\left(1+\Delta_{i}\right)$ is equal to zero if the vectors $k_{1}, \ldots, k_{n+1}$ generate the space $L^{n}$. In the opposite case this function is equal to one.

Proof. The minors $\Delta_{i}, i=1, \ldots,(n+1)$, are the coordinates of the vector product of the vectors $A^{*} v_{1}, \ldots, A^{*} v_{n}$ (rows of the matrix composed of columns $k_{1}, \ldots, k_{n+1}$ ). The vectors generate the space if and only if at least one of the minors $\Delta_{i}$ is not equal to zero (and therefore it is equal to one).

Lemma 5. The following formula is true:

$$
\bar{F}\left(k_{1}, \ldots, k_{n+1}\right)=\left(1+\Delta_{1}+\cdots+\Delta_{n+1}\right)+\prod_{1 \leq i \leq n+1}\left(1+\Delta_{i}\right) .
$$

Proof. If the vectors $k_{1}, \ldots, k_{n+1}$ do not generate the whole space $L^{n}$, then all the minors $\Delta_{i}$ are equal to zero and $\bar{F}=1+1=0$ (everything is happening in the field $\mathbb{Z} / 2 \mathbb{Z}$ ). If the vectors $k_{1}, \ldots, k_{n+1}$ do generate the whole space, then they are related by a unique relation $\sum \Delta_{i} k_{i}=0$. In this case $\prod\left(1+\Delta_{i}\right)=0$ (see lemma 4$)$. By the definition of the function $\bar{F}$ we have

$$
\bar{F}\left(k_{1}, \ldots k_{n+1}\right)=\left(1+\Delta_{1}+\cdots+\Delta_{n+1}\right),
$$

just as was required to prove. 


\section{$\S 10$. Polyhomomorphisms and representation of functions of a parallelepiped through functions of its vertices}

Is it possible to represent a function of a parallelepiped as a linear combination of functions of its vertices? In the general case, the answer is, of course, negative, but sometimes it is possible to do so. For us it is especially important that it is possible to do so for the function which assigns to the parallelepiped $\Delta=I_{1}+\cdots+I_{n}$ the product of roots of the system of binomial equations whose Newton polyhedra are the segments $I_{1}, \ldots, I_{n}$. In this paragraph we will first discuss the general question of polarization of a map $f: G^{n} \rightarrow G_{1}$ from a cartesian power of one abelian group to another abelian group. Then we are going to apply the result to the question in hand.

The map $f: G^{n} \rightarrow G_{1}$ from the direct sum $G^{n}=G+\cdots+G$ of $n$ copies of the abelian group $G$ to the abelian group $G_{1}$ will be called a polyhomomorphism if for every $i, 1 \leq i \leq n$, and every fixed collection $\left\{a_{j}\right\}$ of elements in the group $G, i \neq j$, $1 \leq j \leq n$, the restriction $h(x)=f\left(a_{1}, \ldots, a_{i-1}, x, a_{i+1}, \ldots, a_{n}\right)$ of the map $f$ on the $i$-th component is a homomorphism, i.e. $h(x+y)=h(x)+h(y)$. Multilinear maps between linear spaces are examples of polyhomomorphisms. In this section we give a description of polyhomomorphisms in terms of difference equations.

Let us consider the following operators in the space of maps from $n$-th power of the group $G$ to the group $G_{1}$.

The shift operator $L_{a}^{i}$ in the $i$-th coordinate by a fixed element $a \in G$. This operator translates the map $\varphi\left(x_{1}, \ldots, x_{n}\right)$ to the map $\varphi\left(x_{1}, \ldots, x_{i-1}, x_{i}+a, x_{i+1}, \ldots, x_{n}\right)$. The shift operators for different elements $a$ and indices $i$ commute among themselves. The relation $L_{a+b}^{i}=L_{a}^{i}+L_{b}^{i}$ is true.

The finite difference operator $D_{a}^{i}$ in the $i$-th coordinate. This operator is defined by the formula $D_{a}^{i}=L_{a}^{i}-I$, where $I$ is the identity operator.

Lemma 1. The following equality holds:

$$
D_{a+b}^{i}=D_{a}^{i}+D_{b}^{i}+D_{a}^{i} \circ D_{b}^{i} .
$$

Proof. We have:

$$
D_{a}^{i} \circ D_{b}^{i}=\left(L_{a}^{i}-I\right) \circ\left(L_{b}^{i}-I\right)=L_{a+b}^{i}-L_{a}^{i}-L_{b}^{i}+I=D_{a+b}^{i}-D_{a}^{i}-D_{b}^{i} .
$$

Definition. The map $\varphi: G^{n} \rightarrow G_{1}$ will be called polarizable if for every collection of elements $a_{1}, \ldots, a_{n}$ the map $D_{a_{1}}^{1} \circ \cdots \circ D_{a_{n}}^{n} \varphi$ translates the whole group $G^{n}$ in the same element of the group $G_{1}$. The map $f: G^{n} \rightarrow G_{1}$ defined by the formula $f\left(a_{1}, \ldots, a_{n}\right)=D_{a_{1}}^{1} \circ \cdots \circ D_{a_{n}}^{n} \varphi$ will be the polarization of the polarizable map $\varphi$.

We will be interested in the following problem.

Problem. Is the given map $f: G^{n} \rightarrow G_{1}$ a polarization of some polarizable map $\varphi$ ? If it is, then find all the maps $\varphi$ for which this is true.

The following theorem gives the answer to the question above.

Theorem 1. The map $f: G^{n} \rightarrow G_{1}$ is a polarization of some polarizable map $\varphi: G^{n} \rightarrow G_{1}$ if and only if the map $f$ is a polyhomomorphism and the map $\varphi$ is of the form

$$
\varphi=f+\sum_{1 \leq i \leq n} \varphi_{i}
$$


where $\varphi_{i}$ is any map from $G^{n}$ to $G_{1}$ which does not depend on the $i$-th coordinate. Proof. 1. If the map $\varphi: G^{n} \rightarrow G_{1}$ is polarizable, then its polarization is a polyhomomorphism. Indeed, let us show, for example, that the map $h(x)=D_{x}^{1} \circ D_{a_{2}}^{2} \circ$ $\cdots \circ D_{a_{n}}^{n} \varphi$ is a homomorphism, i.e. $h(x+y)=h(x)+h(y)$.

We have $h(x+y)=D_{x+y}^{1} \circ \cdots \circ D_{a_{n}}^{n} \varphi$. According to lemma $1, h(x+y)=$ $h(x)+h(y)+D_{x}^{1} \circ D_{y}^{1} \circ D_{a_{2}}^{2} \circ \cdots \circ D_{a_{n}}^{n} \varphi$. By hypothesis the map $\varphi$ is polarizable. Therefore the map $P=D_{y}^{1} \circ D_{a_{2}}^{2} \circ \cdots \circ D_{a_{n}}^{n} \varphi$ is constant, and so $D_{x}^{1} P=0$, as required.

2. If the map $f$ is a polyhomomorphism, then $D_{a_{1}}^{1} \circ \cdots \circ D_{a_{n}}^{n} f=f\left(a_{1}, \ldots, a_{n}\right)$. Indeed, $D_{a_{n}}^{n} f\left(x_{1}, \ldots, x_{n}\right)=f\left(x_{1}, \ldots, x_{n-1}, x_{n}+a_{n}\right)-f\left(x_{1}, \ldots, x_{n-1}, x_{n}\right)=f\left(x_{1}, \ldots, x_{n-1}, a_{n}\right)$. Repeating this argument, we will get the required equality.

3. The polarization of the sum $\sum_{1 \leq i \leq n} \varphi_{i}$, in which the map $\varphi_{i}$ does not depend on the $i$-th coordinate, is identically zero. Indeed, let us check, for example, that the polarization of the map $\varphi_{1}: G^{n} \rightarrow G_{1}$, which does not depend on the first coordinate, is identically equal to zero. We have: $D_{a_{1}} \circ \cdots \circ D_{a_{n}} \varphi_{1}=D_{a_{2}} \circ \cdots \circ$ $D_{a_{n}}\left(D_{a_{1}} \varphi_{1}\right)$. But $\left(D_{a_{1}} \varphi_{1}\right) \equiv 0$ since $\varphi_{1}$ does not depend on the first coordinate. The statement is proved.

4. If the polarization of the map $\varphi$ is identically equal to zero, then $\varphi=\sum_{1 \leq i \leq n} \varphi_{i}$, where the map $\varphi_{i}$ does not depend on the $i$-th coordinate. Indeed, if $D_{a_{1}}^{1} \circ \cdots \circ$ $D_{a_{n}}^{n} \varphi \equiv 0$, then $D_{a_{1}}^{1} \circ \cdots \circ D_{a_{n}}^{n} \varphi(0, \ldots, 0)=0$. The last equality means that $\varphi\left(a_{1}, \ldots, a_{n}\right)+\sum_{I}(-1)^{\# I} \varphi_{I}=0$, where $I$ is a non-empty subset of a segment $(1, \ldots, n)$ of natural numbers; $\# I$ is the number of points in the subset $I$; and $\varphi_{I}\left(x_{1}, \ldots, x_{n}\right)=\varphi\left(y_{1}, \ldots, y_{n}\right)$, where $y_{i}=x_{i}$ if $i \notin I$, and $y_{i}=0$ if $i \in I$. ¿From this it follows that the map $\varphi$ is a linear combination of the maps $\varphi_{I}$, each of which does not depend on coordinates with indices $i \in I$. This concludes the proof of the statement.

The theorem now follows from the steps 1-4.

Let us come back to geometry. Let $I_{1}, \ldots, I_{n}$ be $n$ transversal segments in $\mathbb{R}^{n}$, and let $\Delta=I_{1}+\cdots+I_{n}$ be their Minkowski sum. Each vertex $A$ of the parallelepiped $\Delta$ is the sum of some vertices $A_{i}$ of the segments $I_{i}: A=A_{1}+\cdots+A_{n}$. In each segment $I_{i}$ let us fix one of its vertices $l_{i}$ which we are going to call left; the second vertex $h_{i}$ of this segment will be called right and it has the form $h_{i}=l_{i}+a_{i}$, where $a_{i}$ is the vector from the first vertex to the second.

Suppose that $\varphi$ is any map from a collection of $n$ vectors in the space $\mathbb{R}^{n}$ to an abelian group $G_{1}$.

Lemma 2. The value of the map $D_{a_{1}}^{1} \circ \cdots \circ D_{a_{n}}^{n} \varphi$ on the collection of vectors $l_{1}, \ldots, l_{n}$ is equal to

$$
(-1)^{n} \operatorname{sign} \operatorname{det}\left(a_{1}, \ldots, a_{n}\right) \sum_{A \in \Delta} C_{A} \varphi\left(A_{1}, \ldots, A_{n}\right),
$$

where the summation is conducted over all the vertices $A=A_{1}+\cdots+A_{n}$ of the parallelepiped $\Delta$.

Proof. The value of the map $D_{a_{1}}^{1} \circ \cdots \circ D_{a_{n}}^{n} \varphi$ on the collection of vectors $l_{1}, \ldots, l_{n}$ is clearly equal to

$$
\sum_{A \in \Delta}(-1)^{L(A)} \varphi\left(A_{1}, \ldots, A_{n}\right),
$$


where $L(A)$ is the number of left vertices of the segments $I_{1}, \ldots, I_{n}$ among the points $A_{1}, \ldots, A_{n}$ such that $\sum A_{i}=A$. For every vertex $A$ the equality $H(A)+L(A)=n$ holds, where $H(A)$ is the number of right vertices of the segments $I_{1}, \ldots, I_{n}$ among the points $A_{1}, \ldots, A_{n}$ such that $\sum A_{i}=A$. Lemma 2 now follows from theorem 1 in $\S 1$ where we calculated the combinatorial coefficients of a collection of $n$ transversal segments.

Let us fix an integral vector $k \in \mathbb{Z}^{n}$. For any $n$ integral vectors $k_{1}, \ldots, k_{n}$ let us denote by $F_{k}\left(k_{1}, \ldots, k_{n}\right)$ the element $F\left(k_{1}, \ldots, k_{n}, k\right)$ in the field $\mathbb{Z} / 2 \mathbb{Z}$ (see $\S 9$ ). Let us consider the parallelepiped $\Delta$ in $\mathbb{R}^{n}$ with integral vertices.

Definition. The $F_{k}$-volume of the integral parallelepiped $\Delta$ is the following number $F_{k}(\Delta)$. Let us fix any vertex $Q$ of the polyhedron $\Delta$ and denote by $Q_{1}, \ldots, Q_{n}$ the $n$ neighbouring vertices of this parallelepiped. By the $F_{k}$-volume $F_{k}(\Delta)$ of the integral parallelepiped $\Delta$ we will call the element in the field $\mathbb{Z} / 2 \mathbb{Z}$ which is equal to $F_{k}\left(Q_{1}-Q, \ldots, Q_{n}-Q\right)$.

The element $F_{k}(\Delta)$ is well-defined, i.e. it does not depend on the choice of the vertex $Q$ and the order of a neighbouring vertex. Indeed, the function $F_{k}$ does not change under a permutation of vectors or multiplication of certain vectors by $(-1)$ (exactly as the function the absolute value of the determinant).

Lemma 3. The $F_{k}$-volume of an integral parallelepiped $\Delta=I_{1}+\cdots+I_{n}$, where $I_{i}$ are transversal integral segments, is computed by the following formula:

$$
F_{k}(\Delta) \equiv \sum_{A \in \Delta}(-1)^{n} C_{A} F_{k}\left(A_{1}, \ldots, A_{n}\right) \quad \bmod 2 \equiv \sum_{A \in \Delta} F_{k}\left(A_{1}, \ldots, A_{n}\right) \quad \bmod 2 .
$$

Proof. The function $\varphi$ which assigns to $n$ vectors $k_{1}, \ldots, k_{n}$ in the space $\mathbb{Z}^{n} / 2 \mathbb{Z}^{n}$ the element $F_{k}\left(k_{1}, \ldots, k_{m}\right)$ of the field $\mathbb{Z}^{n} / 2 \mathbb{Z}^{n}$ is a multilinear map. According to theorem 1 the map $D_{a_{1}}^{1} \circ \cdots \circ D_{a_{n}}^{n} \varphi$ is constant and is equal to $F_{k}\left(a_{1}, \ldots, a_{n}\right)$. According to lemma 2 we have

$$
F_{k}(\Delta)=(-1)^{n} \sum_{A \in \Delta} C_{A} F_{k}\left(A_{1}, \ldots, A_{n}\right) \bmod 2
$$

as required to prove.

Now we can rewrite the answer in the theorem 1 from $\S 8$. It will be useful to change the notation a bit as well. In $\left(\mathbb{C}^{*}\right)^{n}$ let us consider the system of binomial equations

$$
\begin{gathered}
P_{1}\left(q_{1}\right) x^{q_{1}}+P_{1}\left(r_{1}\right) x^{r-1}=0, \\
\vdots \\
P_{n}\left(q_{n}\right) x^{q_{n}}+P_{n}\left(r_{n}\right) x^{r_{n}}=0,
\end{gathered}
$$

in which $P_{i}\left(q_{i}\right)$ and $P_{i}\left(r_{i}\right)$ are nonzero complex numbers. Let us denote by $I_{i}$ the segment with vertices $q_{i}$ and $r_{i}$. (In the formula below we will denote by the symbol $A_{i}$ any of the vertices $q_{i}, r_{i}$ of the segment $I_{i}$, and we will denote by the symbol $P_{i}\left(A_{i}\right)$ its corresponding combinatorial coefficient $P_{i}\left(q_{i}\right)$ or $P_{i}\left(r_{i}\right)$.) Let us denote by $\Delta$ the Minkowski sum of the segments $I_{1}, \ldots, I_{n}$. 
Theorem 2. Suppose that the parallelepiped $\Delta$ has the full dimensionality, i.e. $n=\operatorname{dim} \Delta$. Then the value of the character $\chi_{k}$ on the product of roots $a_{j}$ of the system (1) is computed by the following formula:

$$
\begin{gathered}
\chi_{k}\left(\prod\left(a_{j}\right)\right)= \\
\prod_{A \in \Delta}\left((-1)^{F\left(A_{1}, \ldots, A_{n}, k\right)} P_{1}\left(A_{1}\right)^{-\operatorname{det}\left(k, A_{2}, \ldots, A_{n}\right)} \ldots\right. \\
\left.P_{n}\left(A_{n}\right)^{-\operatorname{det}\left(A_{1}, \ldots, A_{n-1}, k\right)}\right)^{(-1)^{n} C_{A}},
\end{gathered}
$$

where the product is conducted over all the vertices $A$ of the parallelepiped $\Delta$, $A=A_{1}+\cdots+A_{n}, A_{i} \in I_{i}$; and $C_{A}$ is the combinatorial coefficient at the vertex $A$.

Proof. The required formula can be obtained by rewriting the formula from theorem 1 in $\S 8$ using lemma 3.

\section{$\S 11$. Product of roots of a system of equations with general Newton polyhedra}

In this paragraph we finish solving the problem of calculating the product of roots of a system of equations with a developed collection of Newton polyhedra. The answer is given in two completely dissimilar forms. In the first one the answer is expressed as a product of certain expressions which are called Parshin symbols. The product is taken over all the vertices of the sum of Newton polyhedra which correspond to the equations of the system. Every Parshin symbol enters in the product in the power equal, up to the sign, to the combinatorial coefficient of the corresponding vertex. In the second form, the answer, up to signs, is expressed as the product of a system of coefficients corresponding to the Laurent polynomials; the product is taken over all the vertices of all the Newton polyhedra. Every coefficient of a Laurent polynomial enters in the product in the vector power equal, up to multiplication by $(-n !)$, to the derivative of the mixed volume of the Newton polyhedra by the corresponding vertex.

Suppose that

$$
P_{1}=\cdots=P_{n}=0
$$

is a system of equations in $\left(\mathbb{C}^{*}\right)^{n}$ for which the collection of Newton polyhedra $\Delta_{1}, \ldots, \Delta_{n}$ is developed. Let $\Delta=\Delta_{1}+\cdots+\Delta_{n}$ be the sum of these polyhedra. Let us fix a character $\chi_{k}:\left(\mathbb{C}^{*}\right)^{n} \rightarrow \mathbb{C}^{*}$ and let us compute the value of the character $\chi_{k}$ on the point $M\left(P_{1}, \ldots, P_{n}\right)$, the product of all the roots of the system of equations (1).

We will need the following definition.

Definition. For every vertex $A$ of the polyhedron $\Delta$ we define the Parshin-Kato symbol $\left[P_{1}, \ldots, P_{n}, \chi_{k}\right]_{A}$ of the Laurent polynomials $P_{1}, \ldots, P_{n}$ and the character $\chi_{k}$ in the following way. Let $A=A_{1}+\cdots+A_{n}$ be the decomposition of the vertex $A \in \Delta, A_{i} \in \Delta_{i}$. Define

$$
\begin{aligned}
& {\left[P_{1}, \ldots, P_{n}, \chi_{k}\right]_{A}=} \\
& \quad=(-1)^{F\left(A_{1}, \ldots, A_{n}, k\right)}\left(P_{1}\left(A_{1}\right)\right)^{-\operatorname{det}\left(k, A_{2}, \ldots, A_{n}\right)} \ldots\left(P_{n}\left(A_{n}\right)\right)^{-\operatorname{det}\left(A_{1}, \ldots, A_{n-1}, k\right)} .
\end{aligned}
$$


Theorem 1. For the system of equations (1) the value of the character $\chi_{k}$ on the product $M\left(P_{1}, \ldots, P_{n}\right)$ of roots of the system is computed by

$$
\chi_{k}\left(M\left(P_{1}, \ldots, P_{n}\right)\right)=\prod_{A \in \Delta}\left(\left[P_{1}, \ldots, P_{n}, \chi_{k}\right]_{A}\right)^{(-1)^{n} C_{A}},
$$

where the product is conducted over all the vertices $A$ of the polyhedron $\Delta=$ $\Delta_{1}+\cdots+\Delta_{n}$, and $C_{A}$ is the combinatorial coefficient at the vertex $A$.

Proof. Let us use theorem 2 in $\S 7$. We have the equality

$$
M\left(P_{1}, \ldots, P_{n}\right)=\prod_{s \in S} M\left(T_{1}\left(\Gamma_{1}(s)\right), \ldots, T_{n}\left(\Gamma_{n}(s)\right)\right) .
$$

Theorem 1 is already proven for binomial systems of equations (see theorem 2 in $\S 10)$. Let us use this fact.

$$
\begin{aligned}
\chi_{k}\left(\prod _ { s \in S } M \left(T_{1}\left(\Gamma_{1}(s)\right), \ldots,\right.\right. & \left.\left.T_{n}\left(\Gamma_{n}(s)\right)\right)\right)= \\
& =\prod_{s \in S} \prod_{A \in \Gamma(s)}\left[T_{1}\left(\Gamma_{1}(s)\right), \ldots, T_{n}\left(\Gamma_{n}(s)\right), \chi_{k}\right]_{A}^{(-1)^{n} C_{A}(\Gamma, S)},
\end{aligned}
$$

where the inner product is conducted over all the vertices $A$ of the parallelepiped $\Gamma(s)$. Furthermore, for every vertex $A$ of the partition $\mathcal{R}(\Delta)$ the symbol $\left[T_{1}\left(\Gamma_{1}(s)\right), \ldots, T_{n}\left(\Gamma_{n}(s)\right), \chi_{k}\right]$ does not depend on the choice of the polyhedron $\Gamma(s)$ containing the vertex $A$. Let us denote this symbol by $\left[T_{1}, \ldots, T_{n}, \chi_{k}\right]_{A}$. We have

$$
\chi_{k}\left(M\left(P_{1}, \ldots, P_{n}\right)\right)=\prod_{A \in \mathcal{R}(\Delta)}\left[T_{1}, \ldots, T_{n}, \chi_{k}\right]_{A}^{(-1)^{n} \sum_{A \in \Gamma \subset \mathcal{R}(\Delta)} C_{A}(\Gamma, S)} .
$$

From theorem 3 and theorem 4 in $\S 3$ it follows that $\sum_{A \in \Gamma \subset \mathcal{R}(\Delta)} C_{A}(\Gamma, S)=0$ for all the vertices $A$ in the partition $\mathcal{R}(\Delta)$ which are not the vertices of the polyhedron $\Delta$, and $\sum_{A \in \Gamma \subset \mathcal{R}(\Delta)} C_{A}(\Gamma, S)=C_{A}$ for all the vertices $A$ of the polyhedron $\Delta$. Therefore

$$
\chi_{k}\left(M\left(P_{1}, \ldots, P_{n}\right)\right)=\prod_{A \in \Delta}\left[T_{1}, \ldots, T_{n}, \chi_{k}\right]_{A}^{(-1)^{n} C_{A}}
$$

To conclude the proof we only need to remark that at every vertex $A$ of the polyhedron $\Delta$ by definition we have the equality

$$
\left[T_{1}, \ldots, T_{n}, \chi_{k}\right]_{A}=\left[P_{1}, \ldots, P_{n}, \chi_{k}\right]_{A} .
$$

The theorem is proven.

Let us now rewrite the formula for the element $M\left(P_{1}, \ldots, P_{n}\right)$ in a completely different form. For this we will need the notion from $\S 4$ of the derivatives of the mixed volume by the vertices of the polyhedra. Let us turn to definition of the necessary notions. 
The Newton polyhedra are located in the space $\mathbb{R}^{n}$ of characters of the group $\left(\mathbb{C}^{*}\right)^{n}$; to every integral point $k \in \mathbb{R}^{n}$ corresponds the character $\chi_{k}:\left(\mathbb{C}^{*}\right)^{n} \rightarrow \mathbb{C}^{*}$ which maps the point $x$ to the number $x^{k}$. The dual space $\left(\mathbb{R}^{n}\right)^{*}$ to the space $\mathbb{R}^{n}$ is the space of one-parameter subgroups in the group $\left(\mathbb{C}^{*}\right)^{n}$; to every integral point $m=m_{1}, \ldots, m_{n}$ corresponds the one-parameter subgroup $t^{m}: \mathbb{C}^{*} \rightarrow\left(\mathbb{C}^{*}\right)^{n}$ which assigns to every nonzero number $t$ the point $x=x_{1}, \ldots, x_{n}$, where $x_{1}=$ $t^{m_{1}}, \ldots, x_{n}=t^{m_{n}}$.

For a developed collection of integral polyhedra $\Delta_{1}, \ldots, \Delta_{n}$ every derivative $n ! d_{A_{i}} \operatorname{Vol}\left(\Delta_{1}, \ldots, \Delta_{n}\right)$ is an integral covector on the space $\mathbb{R}^{n}$. Therefore to the derivative $n ! d_{A_{i}} \operatorname{Vol}\left(\Delta_{1}, \ldots, \Delta_{n}\right)$ corresponds a one-parameter group in the space $\left(\mathbb{C}^{*}\right)^{n}$. For every nonzero complex number $t \neq 0$ the element $t^{n ! d_{A_{i}} \operatorname{Vol}\left(\Delta_{1}, \ldots, \Delta_{n}\right)}$ in the group $\left(\mathbb{C}^{*}\right)^{n}$ is defined. In the new version of the formula for the element $M\left(P_{1}, \ldots, P_{n}\right)$ we will use these notations.

For a developed collection of integral polyhedra $\Delta_{1}, \ldots, \Delta_{n}$ let us define the element $M_{0}\left(\Delta_{1}, \ldots, \Delta_{n}\right)$ of the group $\left(\mathbb{C}^{*}\right)^{n}$ as the product of roots of the system of equations $P_{1}=\cdots=P_{n}=0$ such that

1) the polyhedron $\Delta_{i}$ is the Newton polyhedron of the Laurent poynomial $P_{i}$;

2) all the coefficients $a_{i k}$ of the Laurent polynomial $P_{i}=\sum a_{i k} x^{k}$ at monomials $x^{k}$, which correspond to the vertices $k$ of the polyhedron $\Delta_{i}$, are equal to one. From theorem 1 we can see that the element $M_{0}\left(\Delta_{1}, \ldots, \Delta_{n}\right)$ is defined correctly. From the same theorem we can see that $M_{0}^{2}\left(\Delta_{1}, \ldots, \Delta_{n}\right)=\mathbf{1}$. Now let us define the element $M_{1}\left(P_{1}, \ldots, P_{n}\right)$ of the group $\left(\mathbb{C}^{*}\right)^{n}$ by the formula $M_{1}\left(P_{1}, \ldots, P_{n}\right)=$ $M\left(P_{1}, \ldots, P_{n}\right) M_{0}^{-1}\left(\Delta_{1}, \ldots, \Delta_{n}\right)$.

Theorem 2. For every system of equations $P_{1}=\cdots=P_{n}=0$ with the developed collection of Newton polyhedra $\Delta_{1}, \ldots, \Delta_{n}$, the element $M_{1}\left(P_{1}, \ldots, P_{n}\right)$ of the group $\left(\mathbb{C}^{*}\right)^{n}$ is defined by the formula

$$
M_{1}\left(P_{1}, \ldots, P_{n}\right)=\prod_{1 \leq i \leq n} \prod_{A_{i} \in \Delta_{i}} P_{i}\left(A_{i}\right)^{-n ! d_{A_{i}} \operatorname{Vol}\left(\Delta_{1}, \ldots, \Delta_{n}\right)},
$$

where the inner product is conducted over all the vertices $A_{i}$ of the polyhedra $\Delta_{i} ; P_{i}\left(A_{i}\right)$ is the number equal to the coefficient of Laurent polynomial $P_{i}$ at the monomial corresponding to the vertex $A_{i}$; and $n ! d_{A_{i}} \operatorname{Vol}\left(\Delta_{1}, \ldots, \Delta_{n}\right)$ is a oneparameter subgroup in the group $\left(\mathbb{C}^{*}\right)^{n}$ which corresponds to the derivative of the mixed volume $\operatorname{Vol}\left(\Delta_{1}, \ldots, \Delta_{n}\right)$ by the vertex $A_{i}$.

The element $M_{0}\left(\Delta_{1} \ldots, \Delta_{n}\right)$ is defined by the formula

$$
M_{0}\left(\Delta_{1}, \ldots, \Delta_{n}\right)=\prod_{A \in \Delta} \mathbf{F}\left(k_{1}, \ldots, k_{n}\right)^{C_{A}},
$$

where $\mathbf{F}\left(k_{1}, \ldots, k_{n}\right) \subset\left(\mathbb{C}^{*}\right)^{n}$ is the function defined in $\S 8$; the product is conducted over all the vertices $A$ of the polyhedron $\Delta=\Delta_{1}+\cdots+\Delta_{n}$; and $k_{1}, \ldots, k_{n}$ are the components of the decomposition of the vertex $A$.

Proof. Theorem 2 is a reformulation of theorem 1. For the inference of this reformulation it is enough to use the formula for the mixed volume from theorem $3 \S 4$ and the formula for the components of the point $\mathbf{F}\left(k_{1}, \ldots, k_{n}\right)$ from corollary $1 \S 8$. 


\section{REFERENCES}

[1] D. Bernshtein, The number of roots of a system of equations, Funktsional. Anal. i Prilozhen. 9 (1975), no. 3, 1-4; English transl. in Functional Anal. Appl. 9 (1975), no. 3.

[2] Brylinski J.-L. and McLaughlin D. A., Characreristic classes and multidimensional reciprocity laws, Mathematical Research Letters 3 (1996), 19-30.

[3] I. M. Gelfand, M. M. Kapranov, and A. V. Zelevinsky, Discriminants, resultants, and multidimensional determinants, Birkhäuser, Boston, 1994.

[4] O. Gelfond and A. Khovanskii, Newton polytopes and Grothendieck residues, Dokl. Russian Akad. Nauk 3 (1996), no. 350, 298-300; English transl. in Russian Acad. Sci. Dokl. Math.

[5] Gelfond O.A., Combinatorial Coefficients and the Mixed Volume of Polytopes, Functional Anal. Appl. 30 (1996), no. 3, 207-208.

[6] Khovanskii A.G., Algebra and mixed volumes, A Series of Geometry (Y. D. Burago and V. A. Zalgaller, eds.), vol. 285, Springer-Verlag, Berlin-New York, 1988, pp. 182-207.

[7] A. Khovanskii, Consistent partitions of polytopes and polynomial measures, Topics in Singularity Theory: V.I. Arnold's 60th Anniversary Collection, 1997, pp. 157-166.

[8] Khovanskii A.G., Newton polygons, curves on toric surfaces and Weil theorem, "Uspekhi matematicheskikh nauk" 52 (1997), no. 6, 113-142.

[9] Parshin A.N., Local class field theory, Proceedings of the Steklov Institute of Mathematics (1985), no. 3.

[10] Parshin A.N., Galois cohomology and the Brauer group of local fields, Proceedings of the Steklov Institute of Mathematics (1991), no. 4.

[11] O. Ya. Viro, Real algebraic varieties with prescribed topological properties, Thesis Doct. Math. (to appear in Transl. Math. Monographs, Amer. Math. Soc.), Leningr. Univ., Leningrad, 1983. 